\title{
Generalized Mixed Modeling in Massive Electronic Health Record Databases: What is a Healthy Serum Potassium?
}

\section{Cristian G. Bologa}

University of New Mexico Health Sciences Center

\section{Vernon Shane Pankratz}

University of New Mexico School of Medicine

Mark L Unruh

University of New Mexico School of Medicine

Maria Eleni Roumelioti

University of New Mexico School of Medicine

\section{Vallabh Shah}

University of New Mexico School of Medicine

\section{Saeed Kamran Shaffi}

University of New Mexico School of Medicine

\section{Soraya Arzhan}

University of New Mexico School of Medicine

John Cook

Singular Value Consulting, LLC

Christos Argyropoulos ( $\square$ argchris@hotmail.com )

University of New Mexico School of Medicine https://orcid.org/0000-0002-9679-7805

\section{Technical advance}

Keywords: Generalized Linear Mixed Models, Laplace Approximation, Adaptive Gaussian Hermite Quadrature, Electronic Health Records, Dyskalemias, Markov Chain Monte Carlo

Posted Date: February 23rd, 2021

DOI: https://doi.org/10.21203/rs.3.rs-245946/v1

License: (1) (1) This work is licensed under a Creative Commons Attribution 4.0 International License.

Read Full License 
Version of Record: A version of this preprint was published at BMC Medical Research Methodology on July 24th, 2021. See the published version at https://doi.org/10.1186/s12874-021-01318-6. 


\section{Title:}

2 Generalized Mixed Modeling in Massive Electronic Health Record Databases: what is a healthy serum

3 potassium?

4

5 Authors:

6 Cristian G. Bologa ${ }^{*}$, Vernon Shane Pankratz*, Mark L Unruh*, Maria Eleni Roumelioti ${ }^{*}$, Vallabh Shah", ,

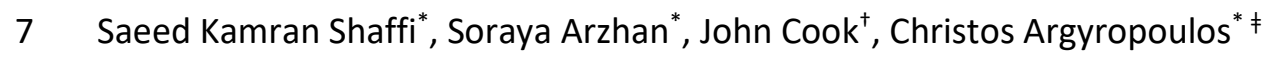

$8 \quad$ *Department of Internal Medicine, University of New Mexico School of Medicine, MSC10 5550

91 University of New Mexico Albuquerque, NM 87131, USA

10 I Department of Biochemistry and Molecular Biology, University of New Mexico School of Medicine

11 MSC08 46701 University of New Mexico Albuquerque, NM 87131

12 + Singular Value Consulting

13 キ Corresponding author: cargyropoulos@salud.unm.edu

14 


\section{Abstract:}

16 Background: Converting electronic health record (EHR) entries to useful clinical inferences requires one

17 to address the poor scalability of existing implementations of Generalized Linear Mixed Models (GLMM)

18 for repeated measures. The major computational bottleneck concerns the numerical evaluation of

19 multivariable integrals, which even for the simplest EHR analyses may involve millions of dimensions

20 (one for each patient). The hierarchical likelihood (h-lik) approach to GLMMs is a methodologically

21 rigorous framework for the estimation of GLMMs that is based on the Laplace Approximation (LA),

22 which replaces integration with numerical optimization, and thus scales very well with dimensionality.

23 Methods: We present a high-performance implementation of the h-lik for GLMMs in the R package TMB.

24 Using this approach, we examined the relation of repeated serum potassium measurements and survival

25 in the Cerner Real World Data (CRWD) EHR database. Analyzing this data requires the evaluation of an

26 integral in over 3 million dimensions, putting this problem beyond the reach of conventional approaches.

27 We also assessed the scalability and accuracy of LA in smaller samples of 1 and $10 \%$ size of the full dataset

28 that were analyzed via the a) original, interconnected Generalized Linear Models (iGLM), approach to h-

29 lik, b) Adaptive Gaussian Hermite (AGH) and c) the gold standard of Markov Chain Monte Carlo (MCMC)

30 for multivariate integration.

31 Results: Random effects estimates generated by the LA were within $10 \%$ of the values obtained by the

32 iGLMs, AGH and MCMC techniques. The H-lik approach was 4-30 times faster than AGH and nearly 800

33 times faster than MCMC. The major clinical inferences in this problem are the establishment of the

34 non-linear relationship between the potassium level and the risk of mortality, as well as estimates of the

35 individual and health care facility sources of variations for mortality risk in CRWD.

36 Conclusions: We found that the combination of the LA and AD offers a computationally efficient,

37 numerically accurate approach for the analysis of extremely large, real world repeated measures data 
38 via the $h$-lik approach to GLMMs. The clinical inference from our analysis may guide choices of

39 threatment thresholds for treating potassium disorders in the clinic.

40

41 Keywords: Generalized Linear Mixed Models, Laplace Approximation, Adaptive Gaussian Hermite

42 Quadrature, Electronic Health Records, Dyskalemias, Markov Chain Monte Carlo 


\section{Background:}

44 Electronic Health Records (EHR) have been adopted near universally in medical practices across the

45 United States. They contain information about vital signs, lab results, medical procedures, diagnoses, medications, admissions, healthcare facility features (e.g., type, location and practice) and individual

47 subject outcomes (e.g., death or hospitalizations). Due to the large number of patients, and the equally large number of features collected in each subject, EHR "big data" is increasingly being mined hoping to gain useful clinical insights that enhance patient safety, improve health care quality and even manage costs [1-4]. A unique feature of EHR data that complicates analytics is the repeated measures and

51 unbalanced nature of the data, featuring multiple and unequal number of observations in individual

52 patients (IP), seen by different healthcare practitioners (HCPs) across healthcare facilities (HCFs). However existing approaches to big data such as deep learning[5] overlook this feature of EHR data, missing on a unique opportunity to mine variation at the level of IP/HCPs or HCFs. Subject or healthcare facility focused inference requires the deployment of conventional Generalized Linear Mixed Models (GLMM) for the analyses of repeated measures at the group (IP/HCP/HCF) level. GLMMs can tackle the

57 entire spectrum of questions that a clinical researcher would want to examine using EHR data. Such questions typically involve the analyses of continuous (e.g., biomarker values, vital signs), discrete (e.g., development of specific diagnoses), time to event (e.g., survival) or joint biomarker-discrete/time-event data.

61 Addressing these questions with GLMMs necessitates the evaluation of high-dimensional integrals,

62 which even for the simplest EHR analyses may involve millions of dimensions (e.g., one for each patient).

63 The implementation of GLMMs in existing statistical environments (e.g. SAS, R) has been shown to scale 64 poorly, when repeated measures for more than a few thousand individuals are analyzed [6, 7]; due to the "curse of dimensionality" numerical integration is no longer tractable either analytically or 
application of formal statistical methods to big datasets. Approximate methods for fitting large GLMMs within the computational constraints of standard multicore workstations [6-8] or even parallel architectures (e.g. LinkedIn's GLMix[9]) have been proposed in the literature. Most commonly analyses rely on specific features of the datasets to speed up calculations through data partitioning and metaanalytic techniques[6]. Other proposals revisit older approaches to statistical inference by applying the method of moments during estimation [7], fitting the models through summary statistics[8] and revisiting unsophisticated but rather robust optimization methods such as coordinate block relaxation and the more recent reincarnation of stochastic gradient descent. Empirical analyses [7] demonstrates that such approaches invariably trade statistical efficiency for speed, effectively discarding valuable information hidden in big data. Finally, quantification of uncertainty about model estimates becomes extremely challenging theoretically and, in some cases, it is not addressed at all in publications (as in 78 GLMix). The ideal approach to the analyses of EHR big data with GLMMs, would seek to eliminate or reduce the need for statistically inefficient approximations, while retaining the rigor, numerical precision, and uncertainty quantification measures (e.g., standard errors) that one has come to expect and trust from analyses of small to medium sized data. In this work we show how these ideals can be retained in big data analytics, by deploying theoretically rigorous estimation methods for GLMMs in a

84 computationally efficient manner. The theoretical background for achieving these goals is based on the Hierarchical likelihood ( $h$-lik) approach to GLMM estimation[10-12]. The $h$-lik was initially introduced as a theoretical framework for understanding the statistical properties of GLMMs, and later received

87 attention for numerical work. In the context of $h$-lik, the Laplace approximation (LA) is used to replace 88 multivariate integration with optimization, paving the road for computationally efficient implementations that are uniquely suited to tackle the challenges posed by EHR big data. However this 
91 interconnected General Linear Model (iGLM) formulation [12] which can be challenging to correctly

92 implement[13] and which we may not even for large numbers of random effects as we show below. In

93 this work, we circumvent these limitations by directly implementing the $h$-lik method in the R package

$94 \mathrm{TMB}[14]$. TMB combines the LA for the numerical evaluation of multivariate integrals, with Algorithmic

95 Differentiation (AD) for the fast calculation of objective (log-likelihood) functions. TMB itself implements

96 an approximate estimation method for the marginal likelihood of GLMMs $[15,16]$ but to date has not

97 been adopted to implement $h$-lik calculations. TMB provides the key missing ingredients for fast,

98 scalable $h$-lik calculations: AD, parallel computations to speed up the most computationally challenging

99 steps and built-in uncertainty quantification features.

100 Motivating example

101 The analyses reported in this paper were motivated by the following clinical considerations from the

102 physician author's subspecialty (nephrology):

103 - Abnormalities in the serum potassium levels ( $\mathrm{K}^{+}$, dyskalemia), i.e., either low (hypokalemia) or high

104 (hyperkalemia) are commonly encountered in clinical practice[17]. Aberrations in serum potassium

105 will in turn interfere with the electrical conduction and cell membrane function in many tissues,

106 including muscle, bowel and heart leading to muscle weakness/small bowel ileus, cardiac

107 arrhythmias and even death.

108 - The precise relationship of $\mathrm{K}^{+}$with mortality across the spectrum of kidney function, remains poorly

109 defined. The largest to date repeated measures study in the US examined a cohort of 55,266

110 individuals from a single Managed Care organization in California[18]. It demonstrated a "U" shaped

111 curve suggesting that mortality was higher for both hypokalemia and hyperkalemia. However, this

112 analysis was limited in examining outcomes from a single health care organization and discretized

113 the $\mathrm{K}^{+}$measurements prior to analysis. Therefore, a continuous risk relationship that generalizes

114 across the entire United States is difficult to infer from this publication. 
- This lack of precision limits rational use of both potassium replacement in patients with low $\mathrm{K}^{+}$ (hypokalemia) and potassium binding drugs in those with elevated $\mathrm{K}^{+}$(hyperkalemia). Existing and emerging therapies for hypertension[19], congestive heart failure[20-22] and therapies for diabetic kidney disease[23, 24], require the use of drugs e.g. the mineralocorticoid receptor antagonists that will variably elevate the potassium level[25]. By leveraging the EHR we hope to inform future practice guidelines for the management of potassium disorders in clinical practice. For example, these guidelines could link initiation of therapies to treat dyskalemias to the prevailing $\mathrm{K}^{+}$level, using the risk relation identified in real world data to specify treatment thresholds.

\section{Contributions}

These can be summarized as follows:

1) We provide a roadmap for the direct, accurate and scalable implementation of GLMMs using the $h$ lik approach. This implementation effectively maps the core concepts of the statistical theory behind $h$-lik, to calls to software libraries that use the LA to approximate marginal likelihoods. Rather than having to worry about manually encoding a high-performance implementation, the analyst can leave the numerical subtleties to be decided by the library. This implementation is scalable enough to be deployed in standard multicore workstations available to most clinical epidemiologists and as we show below yields answers to clinically meaningful questions in an acceptable timescale. Using real world big data, we show that the results obtained by our implementation are very similar to those obtained via theoretically more accurate techniques for GLMM estimation, i.e. Adaptive Gaussian Hermite (AGH) or Markov Chain Monte Carlo (MCMC) or even the original iGLM approach to the $h$-lik.

2) We demonstrate the deployment of $h$-lik to fit Poisson GLMMs in datasets derived from the Cerner Healthfacts Electronic Health Record (EHR) database (now referred as Cerner Real World Data and abbreviated as "CRWD" in this work). Cerner is one of the major EHR used in the United States. It 
contains laboratory, clinical and outcomes data from nearly a third of US healthcare facilities over a period of more than 10 years.

3) We address the gap in the clinical knowledge about the relation between $\mathrm{K}^{+}$with mortality adjusting for the effect of kidney function on this relation.

4) We quantify the interindividual and healthcare facility variation around the curve that relates the $\mathrm{K}^{+}$ level to survival. Assessment of such variability is a major advantage of GLMMs over other analytical approaches, e.g., deep learning and has implications for both policy and clinical practice.

This paper is organized as follows. In the methods section we introduce CRWD and the dataset used for our analyses. We will also also review $h$-lik for GLMMs, and alternative methodologies for estimating GLMMs (AGH and MCMC), the original (iGLM) approach for fitting the $h$-lik and describe our own implementation of the $h$-lik calculations in the R package TMB. In the results section we compare the results obtained by TMB and the referent GLMM implementation in R using iGLMs, AGH and MCMC.

152 Unlike the direct implementation of the $h$-lik we consider here, these three alternatives have difficulties 153 in running to conclusion within a reasonable time scale when used in big datasets. Therefore, we used 154 small random samples ( $1 \%$ and $10 \%$ ) of the complete dataset to compare our method against competing 155 approaches. After contrasting these alternatives, we will then present our analyses of the clinical 156 scenario focusing on the relative risk of an abnormal $\mathrm{K}^{+}$level adjusting for the prevailing level of renal 157 function (eGFR), as well as individual and facility sources of variation. We conclude by considering the 158 impact of the proposed computational methodology for EHR big data, applications outside the narrow 159 field of EHR analytics, and the path towards fully parallel implementations of the $h$-lik approach for 160 GLMMs. Parts of this work have been presented in preprint [26] and in abstract/poster form presented 161 during the American Society of Nephrology 2018 meeting [27]. 


\section{Methods}

164 Cerner Real World Data.

165 Cerner is a comprehensive source of de-identified, real-world data that is collected as a by-product of 166 patient care from over 700 healthcare facilities across the United States. The relational database

167 contains clinical records with time-stamped information on pharmacy, laboratory, admission, and billing 168 data for over 69 million unique patients. Types of data available include demographics, encounters, 169 diagnoses, procedures, lab results, medication orders, medication administration, vital signs,

170 microbiology, surgical cases, other clinical observations, and health systems attributes. Detailed

171 pharmacy, laboratory, billing, and registration data go back as far as 2000 and contain more than 630

172 million pharmacy orders for nearly 3,500 drugs by generic name and brand. The two largest tables in the

173 database contain more than 4.3 billion laboratory results and 5.6 billion clinical events linked to more

174 than 460 million patient visits. For our analyses, we used the entire CRWD from inception until

175 September $1^{\text {st }}, 2016$ and extracted the first $\mathrm{K}^{+}$measurement that had been obtained within 24 hours of

176 a clinical encounter. We restricted attention to adult (older than 18 -year-old) patients who were not

177 receiving chronic dialysis and had received at least two $\mathrm{K}^{+}$measurements in two clinical encounters.

178 Individuals were observed until the resolution of the clinical encounter for a total of $29,787,791$ days

179 ( 81,610 patient years). After excluding missing data, this dataset included $9,935,812$ observations in

$1803,123,457$ individuals and 327 facilities. During the observation period there were 48,578 deaths,

181 corresponding to an event rate of 59.5/100 patient years. The median/interquartile range number of

182 repeated observations per patient were $2 / 1$ (mean and standard deviations were 3.18/2.72) and only

$18325 \%$ of patients had three or more repeated measurements. The limited number of observations per

184 patient, a-priori disadvantages the LA, since the latter requires many more observations per individual

185 to converge. Most patients in our analyses had observations from a single $\operatorname{HCF}(2,886,375 / 3,123,457$ or

$18692.4 \%)$. Hence, even though most patients were "nested" inside HCF, those who had "crossed" to 
multiple facilities complicate analyses when random effects at both the IP and the HCF facility are considered.

\section{Modeling the risk of dyskalemia in CRWD via Poisson GLMM.}

190 The data $y_{i}$ for these analyses were death events $\left(y_{i}=1\right)$ recorded in CRWD. This outcome was 191 modelled as a Poisson variable, by exploiting the link between Poisson models and analysis of survival 192 time [28], using the duration of each clinical encounter as an offset. Predictors that were coded as fixed 193 effects included the $\mathrm{K}^{+}$level, the Charlson Comorbidity Index, a well validated instrument of the 194 comorbid conditions affecting an individual, the prevailing level of kidney function (estimated

195 Glomerular Rate, eGFR, computed by the validated CKD-Epi formula), patient's age, type of healthcare 196 facility (e.g., hospital, nursing home, outpatient clinic), patient's age, race and gender. Natural splines 197 were used to probe non-linear relationships between the outcome of interest, kidney function (eGFR) 198 and the potassium level as well as age. During the comparative evaluation of methods for fitting the 199 dataset, we run two separate analyses with different random effects specifications: one in which the 200 grouping level was the individual (IP) and one in which the grouping level was the facility (HCF). These 201 analyses allowed us to explore the scaling of performance of various estimation methods for GLMMs 202 against the increasing dimensionality of the problem. The latter is largely determined by the number of 203 random effects, since the vector of fixed effect coefficients is low dimensional, i.e., $p=18$ in all analyses. 204 Clinical inference about the relation between the $\mathrm{K}^{+}$level and the risk of death was based on the full 205 dataset using random effects at both the IP and HCF level.

\section{GLMMs estimation requires high-dimensional integrations.}

207 Let $y_{i}, \mathrm{i}=1 \ldots n$ denote data and let $x_{i}$ and $z_{i}$ stand for vectors of covariates for each data element (IP) 208 in the model. The $x_{i}$ vector is multiplied by the global ("fixed effects") $p$ dimensional coefficient vector $209 \beta$ that models the overall response, while the $z_{i}$ by a group specific, $q$ dimensional vector $u$ of random 210 effects. In the simplest case, the random effects feature vector indicates the group membership of the 
211 data, e.g., which observations came from the same individual, but more complex scenarios are possible

212 dependent on the repeated measures and clustering structure of the data. A GLMM is defined by the

213 following properties:

1. The probability density function of the data $\left(f_{Y \mid U}\right)$, conditional on the random and fixed effects vectors, is a member of the exponential family. The nature of the data determines the exponential family, e.g. for binary classification this would be the binomial distribution, for continuous data the Gaussian distribution, and for the analysis of time-to-event or counts of events (as in the present case) the Poisson distribution[28].

2. The expectation of the data $\mu_{i}$ conditional on the random effects is given by the equation: $\mathrm{g}\left(\mu_{i}\right)=x_{i}{ }^{T} \beta+z_{i}^{\mathrm{T}} u$. The function $\mathrm{g}(\cdot)$ is the link function and is determined by the nature of the regression e.g., it is the logistic map for binary classification, or the logarithmic function when modeling count data. The variance of the data is determined as a product of a dispersion parameter (conventionally indicated as $\phi$ ) and a variance function that is strictly a function of the conditional mean. For the Poisson GLMM we are considering here, the dispersion parameter is equal to one and the variance function is equal to the mean (see Table in p45 of [12] for variance functions of other generalized models). For the remainder, and without loss of generality we will assume that the dispersion parameters are known.

3. The random effects vector is assumed to have a zero mean multivariate normal distribution and covariance matrix $\mathrm{G}=\mathrm{G}(\gamma)$ where $\gamma$ are the variance components, i.e., $f_{U \mid \gamma} \sim \mathrm{N}(0, G)$. We stress that the specification of random effects in the $h$-lik is not restricted to the normal distribution, yet this choice is implicitly made in the comparator AGH methods. 
233 Learning in a GLMM context corresponds to estimating the values of the variance components $(\gamma)$, the

234 fixed $(\beta)$ and the random effects $(u)$. Maximum likelihood estimation (MLE) for the variance components and the fixed effects involves maximization of the marginal likelihood $L(\beta, \gamma)$ :

$$
\max _{\beta, \gamma} L(\beta, \gamma)=\max _{\beta, \gamma} \int \exp \left(\log \mathrm{f}_{Y \mid U}+\log \mathrm{f}_{U \mid \gamma}\right) \mathrm{du}(1)
$$

237 The integral in (1) is typically over the high dimensional space of the random effects and can only be 238 approximated numerically. In particular, evaluation of such integrals in closed form are typically 239 available for only a few combinations of random effects and response distributions. Quadrature approximations replace integrals via weighted sums over predefined abscissas ("nodes") that are placed 241 over the domain of integration. The quality of the approximation and the computational resources 242 required to evaluate the integral will scale up with the number of nodes (order) used by the quadrature 243 rule. However, the curse-of-dimensionality makes the use of higher order (more accurate)

244 approximations impractical: applying an $l$ order rule in $m$ dimensions will require $m^{l}$ evaluations of the 245 integrand $\exp \left(\log \mathrm{f}_{Y \mid U}+\log \mathrm{f}_{U \mid \gamma}\right)$, thus quickly exhausting computational resources. Integration of

246 GLMMs in most statistical computing environments is based on Adaptive Gauss-Hermite

247 quadrature[29-32]. In contrast to its non-adaptive version that places the nodes around zero, AGH 248 carefully centers the location of the nodes by finding the values of the random effects (conditional 249 modes, $\hat{u}$ ) that maximize the function $\log \mathrm{f}_{Y \mid U}+\log \mathrm{f}_{U \mid \gamma}$ and scales them according to the curvature of 250 that function around its mode. These scaling factors are determined from the second derivatives 251 (entries of the Hessian matrix) of $\log f_{Y \mid U}+\log f_{U \mid \gamma}$ around its maximum. While a significant advance in 252 numerical mathematics for GLMMs, the combination of MLE and AGH is not exactly problem free:

253 a. AGH is still as subject to the curse of dimensionality as the non-adaptive versions of Gaussian quadrature: it provides a more efficient way to spend one's fixed computational budget, by focusing on the area of the integrand that is substantially different from zero. For all intents and 
purposes, estimation of GLMMs with large number of random effects will be limited to a single node (AGH1) which is just the Laplace Approximation see ([33-35] and below)

b. The variance components will often be estimated with considerable bias, especially for discrete outcome (e.g. logistic or Poisson) models and few observations per random effect $[13,36]$, leading to proposals that replace MLE with Restricted Maximum Likelihood (REML)

estimation[37, 38]. REML maximizes the integral $\int \exp \left(\log \mathrm{f}_{Y \mid U}+\log \mathrm{f}_{U \mid \gamma}\right) \mathrm{d} u \mathrm{~d} \beta$ and reduces the numerical evaluation of multivariate integrals.

\section{H-likelihood for GLMMs}

The $h$-lik is a statistical procedure for estimating GLMMs, in which the relevant calculations proceed in stages[10, 10, 11, 38, 39]. The starting point for $h$-lik is the joint likelihood of the data i.e., the product of

$270 f_{Y \mid U}$ and $f_{U \mid \gamma}$. The $h$-likelihood function $(h)$ is the logarithm of this product and it is the object used 271 during computations [11] that are based on the function class $p_{w}(l(h))$ defined as:

$$
p_{w}(l(h))=\left[l(h)-\frac{1}{2} \log |-\mathrm{H}(l(h), \widehat{w}) / 2 \pi|\right]_{w=\widehat{w}}(2
$$

273 where $l(h)$ is a functional of the h-likelihood function, $|\cdot|$ is the determinant, $\mathrm{H}(l(h), \widehat{w})$ is the Hessian

274 (matrix of second derivatives) of the function $l(h)$ with respect to the argument $w$, evaluated at the 275 point $\widehat{w}$ verifying the (non-linear) system of the score equations $\partial l(h) / \partial w=0$. The functional $l(h)$ 276 depends on the nuisance parameters $w$, as well as other parameters of inferential interest, i.e., $l(h)=$

$277 l\left(h(q, w)\right.$. In the context of the statistical theory, $p_{w}(l(h))$ is an adjusted profile log-likelihood [40] that 
278 allows the elimination of the nuisance effects $w$, through marginalization (random effects) or

279 conditioning (fixed effects)[11]. These eliminations are required during steps 1-2 of the $h$-lik inferential

280 procedure:

1. Inference about the variance components is based on the conditional density:

$$
\mathrm{y} \mid \hat{\beta}, \gamma \sim \exp (\log L(\beta, \gamma)) / \exp (\log L(\hat{\beta}, \gamma))(3)
$$

with $\hat{\beta}$ the maximizer of the marginal likelihood in (1). This conditional density approximates the REML functional $\iint \exp \left(\log f_{Y \mid U}+\log \mathrm{f}_{U \mid \gamma}\right) \mathrm{d} u \mathrm{~d} \beta[12]$.

2. Inference about the fixed effects is based on the marginal likelihood $L(h)=L(\beta, \gamma)$.

3. Inference about the random effects is based on the $h$-likelihood function $h(\beta, \gamma, u)=\log f_{Y \mid U}+$

While optimization of the h-likelihood function is straightforward, multivariate integrations are required when making inferences about the fixed effects and variance components. Computationally the $h$-lik uses the LA to carry out the relevant integrations for the restricted (3) and marginal likelihoods, thus replacing multivariate integration by the much simpler problem of numerical optimization. For a general $\mathrm{p}$ dimensional integral, the LA for a function in the variables $q, I(q)$, defined via the integral of an exponentiated function $h(q, w)$, over the variables $\mathrm{w}$, is given by:

$$
I(q)=\int \exp (h(q, w)) \mathrm{d} w \approx(2 \pi)^{\mathrm{p} / 2} \exp \left(h\left(q, \widehat{w}_{q}\right)\right)\left|-\mathrm{H}\left(h, \widehat{w}_{q}\right)\right|^{-1 / 2} \equiv \exp \left(p_{w}(h)\right)
$$

295 where $\widehat{w}_{q}$ is the optimum of the function $h(q, w)$ for a given value of $q$ and $\left|-\mathrm{H}\left(h, \widehat{w}_{q}\right)\right|$ is the 296 determinant of the Hessian matrix of the function $h(q, w)$ with respect to $w$, evaluated at the optimum.

297 Direct comparison of (2) and (4) shows that the adjusted profile likelihood used by the $h$-lik is the logarithm of the LA to the integrals defining marginal likelihoods. The relevant profile likelihoods used by $h$-lik to estimate the fixed effects and the variance components are denoted by $p_{u}(h)$ and $p_{u, \beta}(h) \approx$ 
$p_{\beta}(\log L)$ respectively. The predicted values of the random effects may be derived as conditional expectations given the observed data (BLUP: Best Linear Unbiased Predictor). The values of these may

302 also be obtained by optimizing the h-likelihood function $h(\beta, \hat{\gamma}, u)$ given $\beta$ and the estimate of the 303 variance parameters $\hat{\gamma}$ [10]. Applying the LA to compute the $p_{u}(h)$ obtains estimates of the BLUPs for 304 "free".

\section{The interconnected GLM algorithm for H-likelihood estimation}

306 While the theory of the $h$-lik is presented in terms of the LA, the actual numerical computations as

307 introduced [10] and latter extended by numerous authors $[11,12,41]$ is based on an additional 308 approximation. Specifically, an Extended Quasi-Likelihood (EQL) approach [42] is uses to model the 309 mean and the variance of the response variable $(y)$ and the random effects $(u)$. Replacing the original

310 GLM of the response variable and the probability density random effects by an approximation based on 311 their first two moments, would appear a poorly justified choice given the loss of accuracy and potential 312 for bias. However, this choice also allows the analyst to use the deviance (residuals) of a Generalized 313 Linear Model (GLM) as data. This feature of the EQL reduces estimation of fixed, random effects, 314 dispersion parameters and variance components to a clever, iterative fitting algorithm of interconnected 315 GLMs (iGLM, see Chapter 7 in [12] and the documentation of the R implementations[43, 44] ). The steps 316 of this algorithm are:

317 1. Initialize starting values for $\beta, u, \gamma$ and $\phi$ (if the GLM for the response includes a dispersion)

2. Construct an augmented model with $y_{a u g}=\left(\begin{array}{c}y \\ E(u)\end{array}\right)$, by stacking the observed data $y$ and the expected values (mean) of the random effects $E(u)$. In the case of multivariate normal effects these expectations are identically zero.

3. Construct the double EQL approximation to the components $\log f_{Y \mid U}, \log f_{U \mid \gamma}$ of the $h$ likelihood function. 
4. Fit the EQL approximation to the augmented data, by using the Iterative Weighted Least Squares (IWLS) algorithm for estimating GLMs. In this augmented GLM, each element of the augmented response is weighted inversely by a dispersion parameter. The latter is equal to $\phi_{i}$ for $y_{i}$ and to the corresponding diagonal element of the covariance matrix $G(\gamma)$ of the random effects. $d_{i} /\left(1-h_{i}\right)$ and prior weights $\left(1-h_{i}\right) / 2$ for each augmented observation.

8. Iterate steps 4-7 until values no longer change and obtain standard errors using the final 
Variations of this algorithm have been proposed to correct for errors introduced by the various

347 approximations and the dependence of the random effects on dispersion parameters $[12,13,39]$. If

348 correctly implemented, the iGLM approach is very fast to converge, as is typical of block-coordinate

349 optimization algorithms $[45,46]$, in which one keeps certain parameters fixed, while sequentially

350 updating others and cycling through the parameter list until estimates no longer change.

351 Pitfalls in the high performance implementation of the iGLM have been previously described [13] : they

352 include errors in the implementation of the update equations and the derivatives (gradients) for the

353 various GLMs, sparse representations for the matrices appearing in the gradients and updating

354 equations, numerical imprecisions when finite differences are used to calculate the gradients and the 355 large computational burden if finite differencing is used to calculate the Hessian, from which standard

356 errors of estimates are computed. Current implementations of the iGLM algorithms are available in R 357 package hgIm[43] and the (archived) package HGLMMM[44] as well as GenStat. To our knowledge, a 358 scalable, direct implementation of the hierarchical likelihood estimation for GLMMs that is not based on 359 the rather complex, error-prone coding of the iGLM algorithm has not been reported. Our 360 implementation designated as $h$-lik for the remainder of this paper is discussed below.

361 A scalable implementation of the $h$-lik using AD and the LA.

362 For a software implementation of $h$-lik to align with the corresponding methodology, it is only required 363 that inferences about the variance components be based on $p_{u, \beta}(h)$ and for the fixed and random 364 effects on $p_{u}(h)$ and $h$ respectively. To implement $h$-lik for very large datasets, one needs ways to 365 calculate and optimize general functions of the form $p_{w}(h)$ in a manner that scales favorably with 366 problem size. Scalability is afforded by a) bypassing finite differences for gradients during numerical optimization of the adjusted profile likelihoods, b) automatic detection of the sparsity (zero elements) in the Hessian and gradient matrices used in the optimization and c) parallelization of calculations of the 
value of the log-likelihood. The availability of all three features would free the analyst to concentrate on model (or equivalently the joint likelihood) specification and let the computer organize calculations in an

371 efficient, expedient manner.

372 The benefits of $A D$ in this context are clear: a) the gradients used during optimization are computed with

373 numerical accuracy that rivals that of hand-coded analytical expressions b) computational cost for

374 obtaining these gradients is of the same order as evaluating the original function (in contrast to finite

375 differencing, in which the cost increases with the number of dimensions) [47-49] c) optimized software

376 code for the Hessians also becomes available to the analyst at zero cost. The benefit of automatic

377 generation of code for the Hessian is two-fold a) it allows the use of second order optimization methods

378 (e.g., Newton algorithm) and b) provides standard errors for estimated parameters, by computing the

379 Hessian computed at the optimum. For GLMM applications, the Hessian matrix will be a sparse one (i.e., 380 only a few entries will be non-zero). Computer science algorithms such as graph coloring [50,51] can be 381 used to automatically detect the sparsity pattern of the Hessian for each specific dataset analyzed and 382 tremendously speed up calculations and reduce memory requirements by ignoring elements that are 383 zero.

384 Scalable optimization of marginal likelihoods as previously outlined, was initially proposed in the context 385 of MLE (rather than REML) estimation for mixed models $[15,16]$ and provided the initial use case of the $386 \mathrm{R}$ library TMB [14]. Implementation of any statistical model in TMB requires the writing of code in two 387 languages: $\mathrm{C}++$ and $\mathrm{R}$. Using $\mathrm{C}++$ macros, the user specifies the joint log-likelihood, receives parameters, 388 random effects, data, and initial values from $\mathrm{R}$. $\mathrm{R}$ is used to prepare data, generate initial values, 389 generate a computational tape (see below), invoke the optimizer, and post process the results. An 390 analyst can use TMB to correctly implement $h$-lik by following a few simple steps: 
1. Specify the $h$-likelihood function $h(\beta, \gamma, u)$ in $\mathrm{C}++$ and compiles it into a dynamic library. This is done only once since the dynamic library may be used with different data for the same general model. Hence TMB code for GLMMs is reusable across application domains.

2. Generate a computational graph ("tape") of the calculations required to analyze a specific dataset designating parameters as either fixed or random by calling the TMB package from within R. During tape generation, explicit dependencies between the parameters are identified by TMB and code is automatically generated for the evaluation of the gradient of the log-likelihood and its Hessian.

3. Call an optimizer of one's choice which repeatedly evaluates the adjusted profile likelihood and its gradient until convergence (we typically used a constrained quasi Newton method for the variance components and unconstrained quasi Newton for fixed effects). During this optimization, TMB will internally calculate the values of the parameters that are being eliminated by the profile likelihood using a second order optimization method (Newton's algorithm). Constraining the variance components to be greater than zero is typically required only if these parameters are close to the boundary value of zero, otherwise one may use any unconstrained optimization algorithm. Regardless of the choice of the optimizer, the latter must be able to utilize gradients (computed by $A D)$ and fully leverage the potential of our implementation.

4. Runs the report function of $\mathrm{TMB}$ from within $\mathrm{R}$ to generate standard error of all estimates to quantify uncertainty upon convergence. There are various ways to generate these standard errors of variable speed and potential accuracy: a) numerically invert an estimate of the Hessian, obtained in step 3 if a quasi-Newton optimizer (e.g., BFGS) was used for step 3 (this is the approach we followed here), b) numerical (finite differencing) differentiation of the Jacobian and c) multiplication of the Jacobian from the last point prior to convergence with its transpose.

In our direct implementation of the $h$-lik in TMB, steps 2 and 3 must be called twice. First, we integrate over $\beta, u$ by designating both as "random" when generating the tape. Using this tape during 
optimization amounts to optimizing $p_{u, \beta}(h)$ to obtain a point estimate (and a covariance matrix) for the variance components. Then fixing the value of the variance components, one generates the tape a

417 second time designating only $u$ as random. Optimizing this tape, is equivalent to optimizing $p_{u}(h)$ to 418 obtain point estimates and covariance matrices for the fixed and random effects. In the $h$-lik theory, a 419 third optimization conditional on the values of the variance components and the fixed effects would be 420 required to value of the random effects. We forwent this step, by noting that the estimates of $u$ from 421 the LA to $p_{u}(h)$ will often be numerically similar those obtained from the conditional maximization of 422 the hierarchical likelihood. The major bottlenecks in large datasets involve the computation of the 423 determinant of the Hessian e.g., through a sparse Cholesky decomposition in (3) and the evaluation of 424 the joint log-likelihood. In our analyses we used the high performance implementation of the BLAS 425 libraries[52] (MKL ${ }^{\circledast}$ by Intel that are distributed with Microsoft R Open) as suggested by the creators of 426 the TMB package. We also evaluated the speed up afforded by the parallel calculation of the $h$-likelihood 427 function, a feature which is natively supported by TMB in multicore platforms through OpenMP.

428 It has been previously pointed $[11,13,53]$ out that the $h$-lik approach can be viewed as an 429 approximation to a Bayesian analysis that uses non-informative priors for the fixed effects, dispersion 430 parameters (if they are not known) and variance components. This connection allows one to check any 431 method implementing the $h$-lik (e.g., the iGLM or our direct implementation) by comparing its 432 computations against those obtained by MCMC integration of the corresponding Bayesian model. While 433 MCMC may be considered the gold standard for high dimensional integration, it is a much more 434 computationally demanding technique. Hence, while one can apply MCMC to small problems, one will 435 wait for a very long time for MCMC to finish for datasets of the size we are considering. However, one 436 can compare the results in smaller datasets that are representative of the big dataset. Such datasets 437 may be obtained by simple random sampling without replacement from the final dataset that one would 438 like to analyze. 


\section{Comparative evaluations}

440 To compare different approaches ( $\mathrm{AGH}, \mathrm{iGLM}$, and the direct implementation of $h$-lik) for the integration

441 of marginal likelihoods in GLMMs, we randomly sampled our full dataset and created two subsets that

442 included $1 \%$ and $10 \%$ of patients. We used the R package Ime4 [54] for AGH based inference (function

443 glmer) and utilized quadrature with 1, 5 and 9 nodes to explore the tradeoffs incurred between accuracy

444 and speed. Note that the LA is formally equivalent to an AGH with a single quadrature node, so that the

445 comparison between AGH1 and $h$-lik reflect the speedup due to the LA/AD combination. Bayesian

446 analyses were carried out by invoking the Poisson GLMM in the STAN programming language [55] as

447 implemented in the R package rstanarm [56]. Like TMB, STAN codes the statistical model into a C++

448 language template, which is also compiled into a dynamic library that implements the calculations for

449 the posterior probability kernel of the model. In this case, the kernel coincides with the h-likelihood

450 function. STAN also utilizes AD to compute the gradient of the model that are used during MCMC with

451 the No U Turn Sampler [57]. Therefore, the comparison against STAN allows an assessment of the

452 accuracy-speed trade-off of MCMC against the $h$-lik computations when the latter are interpreted as

453 modal approximations to a full Bayesian analysis.

454

$455 \quad$ Hardware and timing

456 Timing information was generated in a high-end consumer workstation equipped with the i7-5960x

457 octacore Intel Processor equipped with $32 \mathrm{~GB}$ of DDR4 RAM clocked at $2133 \mathrm{MHz}$. The base frequency of

458 the $\mathrm{i7}-5960 \mathrm{x}$ is at $3 \mathrm{GHz}$, but the processor was overclocked to $3.8 \mathrm{GHz}$ for the timing experiments. For

459 the analyses of the full dataset, we utilized a Dual 16-core Xeon Gold 5218 clocked at $2.3 \mathrm{GHz}$ (turbo

460 single core frequency of $3.9 \mathrm{GHz}$ ) equipped with 512GB of DDR4 RAM (clocked at 2666M Hz) running

461 Windows 10 Pro for Workstations. Large memory requirements did not allow us to fit the parallel

462 version to the full dataset, even in this large memory machine. For the comparative evaluation of 
execution timing in the smaller datasets we ran the iGLM and the parallel TMB analyses thrice on the i7-

$4645960 x$ and the Xeon. To compare parallel and serial implementations of the $h$-lik in the TMB

465 environment, we ran analyses of the $1 \%$ and $10 \%$ datasets on the $17-5960 x$ using both parallel and serial

466 versions of the code that incorporated the two random effects. In addition, we run all three datasets

467 with the serial code on the dual Xeon. Analysis of variance was used to explore the relative impact of 468 dataset size, parallel vs serial version of the code and computing platform, on execution ("wall") times.

469 We collected timing information for all the steps of the TMB $h$-lik calculation e.g., the generation of the

470 tape, optimization, and calculation of the Hessian at convergence, only the total wall time is reported.

471 All analyses were run using Microsoft R Open v 3.5.3 and v4.02 and TMB package v1.7.14-1.7.15.

472

473 Results:

474 Estimates of $h$-lik against AGH and MCMC based methods for single random effects Poisson models.

475 Analyses of the $1 \%$ and $10 \%$ datasets with glmer generated warnings about at least one non-positive

476 definitive Hessian matrix suggesting that the optimization algorithm got stuck close to the true global

477 optimum. Furthermore, AGH1 failed to converge at all, when the measurements were grouped at the IP

478 level. In contrast to previous reports [6], we could obtain a solution from glmer for the $10 \%$ dataset for

479 very large number of random effects but only if the AGH5 or AGH9 method were used. Convergence

480 failure of the AGH1-9 methods implied that their estimates may not reliable. We thus considered an

481 alternative: a "zero" order method that is also available from within glmer [7]. This method effectively

482 ignores the integral for the random effects and thus its estimates will contain a variable and unknown

483 amount of bias. Nevertheless, this approximation usually converges without warnings as we found in

484 our datasets. The results of the direct implementation of $h$-lik with TMB, AGHO, and AGH9 are shown in

485 Figure 1 as estimate (point) and 95\% confidence interval for the two groupings and the two datasets. In 
general, the estimates of our $h$-lik implementation were numerically similar to the AGH methods,

487 suggesting that the nonconvergence reported by the latter would not affect inferences about the fixed

488 effects. The difference among the different methods lies within the uncertainty of the estimate from

489 each method; in fact, estimates were identical for most covariates up to two significant digits.

490 Nevertheless, there were substantial differences among the methods when the estimate of the single

491 variance component was examined. Table 1 shows the estimates for the direct implementation of $h$-lik

492 in TMB, the iGLM implementation in the R package $h g / m, A G H O$ and AGH9. The variance of the

493 estimates could not be obtained for the AGH methods and thus are not reported. Furthermore, we

494 could not fit the iGLM to any dataset with IP random effects due to large memory requirements (i7-

495 5960x) and numerical errors in platforms not limited by memory (Xeon).

496 Table 1 : Estimates of the standard deviation of the variance components for single random effects 497 Poisson models.

498

\begin{tabular}{|c|c|c|c|c|c|}
\hline Dataset & Group & h-lik & iGLM & AGHO & AGH9 \\
\hline $1 \%$ & Individual & $\begin{array}{c}0.306 \\
(0.289)\end{array}$ & & 0.253 & 0.260 \\
\hline $10 \%$ & Individual & $\begin{array}{c}0.469 \\
(0.074)\end{array}$ & - & 0.505 & 0.504 \\
\hline $1 \%$ & Facility & $\begin{array}{c}0.422 \\
(0.085)\end{array}$ & $\begin{array}{c}0.395 \\
(0.047)\end{array}$ & 0.401 & 0.414 \\
\hline $10 \%$ & Facility & $\begin{array}{c}0.703 \\
(0.061)\end{array}$ & $\begin{array}{c}0.669 \\
(0.042)\end{array}$ & 0.699 & 0.701 \\
\hline
\end{tabular}

499 Increasing the size of the dataset results in a greater agreement among the three methods and

500 reduction in the magnitude of uncertainty in the estimates of the proposed $h$-lik method. Estimates by

501 the AGH5 method were identical to the AGH9 to three significant digits (not shown). Bayesian analyses

502 may be considered the benchmark against which other methods of integration for GLMMs should be

503 judged. However only the $1 \%$ dataset with grouping at the facility level could be fit in reasonable time

504 (see timing below). The Bayesian point estimate (posterior mean) of the variance component was 0.426 
505 (standard deviation 0.089), an estimate that was in rough agreement with the estimates generated by

506 all the methods in Table 2 but was numerically closer to the direct $h$-lik estimate of 0.422 . The Bayesian

507 model estimates (posterior mean and standard deviation) for the fixed effects against the direct $h$-lik

508 and iGLM implementations are shown in Table 2. Point estimates and standard errors returned by the

509 two $h$-lik implementations were in close numerical agreement. Many of the MCMC estimates, including

510 the coefficients for the natural splines used to model the effects of age and eGFR were close to those

511 obtained by the $h$-lik implementations. Notable exceptions were the coefficient for some of the racial

512 groups and the coefficients for the natural splines of the potassium level. Despite the differences in the

513 numerical values of the spline coefficients, the splines estimated by the proposed $h$-lik implementation

514 and the MCMC method were nearly identical (

515 Figure 2).

516

517 Table 2 : Fixed effect estimates, summarized by the mean(standard error) from Bayesian and h-lik (as

518 implemented in this paper and the iGLM algorithm) analyses for the $1 \%$ dataset with grouping at the

519 facility level.

\begin{tabular}{|l|c|c|c|}
\hline \multicolumn{1}{|c|}{ Fixed Effects } & Bayesian (MCMC) & h-lik & iGLM \\
\hline Intercept & $-6.214(0.961)$ & $-6.218(0.994)$ & $-6.168(0.992)$ \\
\hline Potassium (natural spline coefficient 1) & $8.062(1.979)$ & $6.808(2.462)$ & $6.805(2.461)$ \\
\hline Potassium (natural spline coefficient 2) & $-38.03(31.12)$ & $-9.491(40.24)$ & $-10.00(40.23)$ \\
\hline Potassium (natural spline coefficient 3) & $-75.33(61.7)$ & $-18.50(79.78)$ & $-19.51(79.76)$ \\
\hline eGFR (natural spline coefficient 1) & $-0.406(0.255)$ & $-0.43(0.251)$ & $-0.435(0.251)$ \\
\hline eGFR (natural spline coefficient 2) & $-1.872(0.492)$ & $-1.839(0.505)$ & $-1.839(0.505)$ \\
\hline eGFR (natural spline coefficient 3) & $-1.141(0.502)$ & $-1.091(0.509)$ & $-1.089(0.509)$ \\
\hline Age (natural spline coefficient 1) & $0.305(0.332)$ & $0.309(0.329)$ & $0.306(0.329)$ \\
\hline Age (natural spline coefficient 2) & $-0.086(0.938)$ & $-0.227(0.913)$ & $-0.227(0.913)$ \\
\hline Age (natural spline coefficient 3) & $0.924(0.199)$ & $0.906(0.198)$ & $0.902(0.198)$ \\
\hline Male Gender & $0.214(0.093)$ & $0.214(0.093)$ & $0.214(0.093)$ \\
\hline White Race & $0.155(0.143)$ & $0.158(0.146)$ & $0.160(0.145)$ \\
\hline Hispanic Race & $-0.745(0.853)$ & $-0.457(0.723)$ & $-0.452(0.723)$ \\
\hline Native American & $-0.233(0.829)$ & $0.031(0.731)$ & $0.040(0.730)$ \\
\hline Other Race & $-0.618(0.321)$ & $-0.580(0.323)$ & $-0.571(0.322)$ \\
\hline Unknown Race & $-0.347(0.488)$ & $-0.242(0.472)$ & $-0.238(0.471)$ \\
\hline Inpatient status & $2.404(0.198)$ & $2.390(0.194)$ & $2.397(0.194)$ \\
\hline
\end{tabular}




\begin{tabular}{|l|l|l|l|}
\hline Charlson Comorbidity Score & $0.098(0.014)$ & $0.099(0.014)$ & $0.099(0.014)$ \\
\hline
\end{tabular}

521 The predicted random effects for the $1 \%$ dataset grouped at the facility level obtained by all non- Bayesian 522 methods were rather like those obtained by MCMC (

523 Figure 3), as evidenced by the high correlations. Bland Altman plots (lower diagonal panels in

524 Figure 3 ) indicated that the difference between $h$-lik and the other methods were within $10 \%$ of each 525 other.

526 In summary, all methods appear to generate similar estimates for the fixed effects, rather similar

527 estimates of the variance components, with the proposed direct $h$-lik implementation demonstrating 528 the closest numerical agreement to MCMC.

Timing comparisons between $\boldsymbol{h}$-lik and the AGH methods for single random effect models

Figure 4 contrasts the execution times between $h$-lik and the AGH methods in the i7-5960x platform.

532 Irrespective of the size of the dataset and the grouping structure, the $h$-lik implementation was much 533 faster than the AGH implementation in glmer. The smallest speedup was observed for the $10 \%$ dataset 534 with grouping at the IP level (3.7x faster), but the speedup could range up to $32.5 \times$ ( $1 \%$ dataset, 535 grouping at the HCF level). The direct $h$-lik implementation was only slightly slower than the iGLM in the 536 datasets we could analyze with the iGLM. Analysis of Variance (ANOVA) showed that the size of the 537 dataset (F statistic 68.07) and the estimation method (F statistic 18.1) were much stronger predictors of 538 these timing differences than the grouping structure of the data (F statistic 3.68). Examination of the 539 timing of the three steps of the $h$-lik method showed that the greatest amount of time was spent on 540 optimization, e.g., for the $10 \%$ dataset with random effects at the IP level, tape generation took $137 \mathrm{sec}$, 541 optimization $3327 \mathrm{sec}$ and Hessian calculation/uncertainty quantification 1,844 sec. For the $1 \%$ dataset 542 with random effects at the HCF level, the corresponding times were: $8 \mathrm{sec}$ (tape generation), $17 \mathrm{sec}$ 543 (optimization), $1.7 \mathrm{sec}$ (uncertainty quantification). In sharp contrast, Bayesian methods were much 
544 slower than any of the other methods considered. Therefore, our analyses were limited to the $1 \%$

545 dataset at the HCF level. Parallel simulation of 1,000 samples from each of four independent Markov

546 Chains took 20,517 seconds of wall time, thus was nearly three orders of magnitude slower than either

547 of the two $h$-lik implementations.

548 Potassium level, interindividual and interfacility sources of variation in the risk of death in CRWD

549 Predictors of mortality in the CRWD accounting for both IP and HCF variability by a two random effects

550 model and for the various datasets considered here are shown in Table 2. Increasing the dataset size,

551 stabilized model estimates of the (log) relative risk of death associated with each of the covariates.

552 Increasing the dataset size was associated with a substantial decrease in the standard error of these

553 estimates. There was considerable interindividual and (to a larger extent) interfacility variation in the

554 relative risk of death, as evidenced by the magnitude of the variance components in the full CRWD

555 dataset. The point estimate (standard error) was $0.234(0.034)$ and $1.11(0.067)$ for the IP and HCF

556 components, respectively. These estimates imply that individual factors not captured by the covariates

557 considered in Table 1 will be associated with 2.5 -fold variation in individual risk between the $2.5 \%$ and

558 the $97.5 \%$ risk quantiles of patients with the exact same values of these covariates. Similarly, facility

559 level factors are an important source of variation, though in this case the relative risk of an individual

560 evaluated in a facility at thee $97.5 \%$ quantile vs. a facility in the $2.5 \%$ quantile is 77.5 -fold higher.

561 The coefficients of the spline associated with the $\mathrm{K}^{+}$level appear rather different among the three

562 datasets. Nevertheless, the predicted relative risk of death for a given $\mathrm{K}^{+}$level was essentially identical 563 between the $10 \%$ and the $100 \%$ datasets (

564 Figure 5). Utilizing the full (100\%) dataset resulted in a substantial reduction in the uncertainty $(95 \%$

565 pointwise confidence interval) for a given $\mathrm{K}^{+}$level. The relative risk associated was numerically close to

566 one for a broad range of $\mathrm{K}^{+}$between 3.3 to $4.5 \mathrm{meq} / \mathrm{l}$, i.e., in the vicinity of the "normal" level of 4.0

$567 \mathrm{meq} / \mathrm{l}$. 
Table 3 : Fixed effects estimates summarized by the mean(standard error) from the direct $h$-lik

569 implementation for the $1 \%, 10 \%$ and $100 \%$ dataset with random effects at both the IP and HCF levels.

\begin{tabular}{|c|c|c|c|}
\hline Parameter & $1 \%$ & $10 \%$ & $100 \%$ \\
\hline \multicolumn{4}{|c|}{ Fixed Effects } \\
\hline Intercept & $-6.22(0.99)$ & $-5.22(0.31)$ & $-3.21(0.16)$ \\
\hline Potassium (natural spline coefficient 1) & $6.81(2.46)$ & $8.65(0.74)$ & $11.98(0.32)$ \\
\hline Potassium (natural spline coefficient 2) & $-9.49(40.24)$ & $-39.56(12.39)$ & $-77.79(7.97)$ \\
\hline Potassium (natural spline coefficient 3) & $-18.5(79.78)$ & $-74.64(24.57)$ & $-146.92(15.84)$ \\
\hline eGFR (natural spline coefficient 1) & $-0.43(0.25)$ & $-0.66(0.08)$ & $-0.59(0.02)$ \\
\hline eGFR (natural spline coefficient 2) & $-1.84(0.51)$ & $-1.41(0.15)$ & $-1.44(0.05)$ \\
\hline eGFR (natural spline coefficient 3) & $-1.09(0.51)$ & $-0.32(0.15)$ & $-0.26(0.05)$ \\
\hline Age (natural spline coefficient 1 ) & $0.31(0.33)$ & $0.6(0.12)$ & $0.78(0.04)$ \\
\hline Age (natural spline coefficient 2) & $-0.23(0.91)$ & $1.43(0.32)$ & $2.08(0.11)$ \\
\hline Age (natural spline coefficient 3) & $0.91(0.2)$ & $1.1(0.07)$ & $1.22(0.02)$ \\
\hline Male Gender & $0.21(0.09)$ & $0.17(0.03)$ & $0.17(0.01)$ \\
\hline White Race & $0.16(0.15)$ & $0.06(0.05)$ & $0.1(0.02)$ \\
\hline Hispanic Race & $-0.46(0.72)$ & $0.14(0.15)$ & $0.16(0.05)$ \\
\hline Native American & $0.03(0.73)$ & $-0.08(0.2)$ & $0.16(0.06)$ \\
\hline Other Race & $-0.58(0.32)$ & $-0.12(0.09)$ & $0.08(0.03)$ \\
\hline Unknown Race & $-0.24(0.47)$ & $-0.09(0.15)$ & $0.02(0.05)$ \\
\hline Inpatient status & $2.39(0.19)$ & $2.13(0.06)$ & $2.21(0.02)$ \\
\hline Charlson Comorbidity Score & $0.10(0.01)$ & $0.10(0.00)$ & $0.10(0.00)$ \\
\hline
\end{tabular}

\section{Analysis of performance of $h$-lik computations in large datasets with multiple random effects}

572 Total wall time (in log10 space) for triplicate executions of the parallel and serial versions of the proposed 573 implementations of $h$-lik are shown in

574 Figure 6 and clearly illustrate the exponential increase in execution time with the size of the dataset 575 irrespective of the machine used. ANOVA identified the size of the dataset (F statistic 1950.474) and the parallel implementation ( $F$ statistic 78.030) as more influential predictors than the machine executing the code (F statistic 2.694). A linear regression analysis of the data showed that execution on the Xeon was $16 \%$ faster than the $17-5960 \times$ (95\% confidence interval $-38.4 \%$ to $+2.8 \% p=0.114)$. The serial code was $11.5 \%$ faster ( $95 \%$ confidence interval $-25.8 \%$ to $+5.6 \%, p=0.129$ ) slower to execute than the parallel code. In

581 Figure 7 we illustrate the time spent in various activities when running the $h$-lik programs. Optimization of the $p_{u, \beta}(h)$ profile likelihood took the longest, followed closely by optimization of $p_{u}(h)$. These time- 
584 done in parallel. The divergent effects of parallelization on these two tasks, underline the small effect of 585 parallelization on execution speed.

\section{Discussion}

587 In this report we demonstrate the initial feasibility of fitting GLMMs to mine large clinical EHR databases

588 by combining the LA with AD into a direct implementation of the $h$-lik approach to GLMMs. Analysis of

589 very large datasets is an area in which many existing approaches to numerical integration for GLMM,

590 e.g., those based on AGH or MCMC methods, are impractical because they execute slowly for big

591 datasets with large number of random effects. In contrast, the proposed technical advance can reliably

592 and reasonably fast fit such datasets. Our implementation differs from the original iGLM algorithm for

593 fitting the hierarchical likelihood. The direct implementation of adjusted profile likelihoods avoids

594 programming errors in the hand coding of matrix operations, as well as numerical errors when

595 calculating derivatives by finite differences speeding up calculations. There are several implications for

596 future work and practice as noted below.

\section{Random effects modeling in very big datasets}

598 GLMMs are one of the most versatile modeling techniques in statistics. In recent years, it has been

599 noted that numerous models used for the non-parametric, flexible modeling of data can be viewed as

600 special cases of the GLMMs. Such models include penalized spline approaches in statistics, but also

601 "workhorses" of Machine Learning such as kernel based methods [58]. However, the applications of

602 GLMMs to big data to date has been limited by the computational bottlenecks inherent in evaluating the

603 high dimensional integrals that arise in their mathematical formulation. A previous biomedically

604 oriented paper reported difficulties in fitting random effects models with 890,934 random effects using

605 AGH in glmer in 2012. The authors resorted to a "divide and conquer" technique to fit separate models

606 to chunks of this dataset followed by meta-regression to synthesize evidence from the smaller datasets. 
However, because of their approximative nature, any "divide and conquer" technique will be

608 accompanied by a non-negligible amount of bias. Furthermore, certain approximation approaches may exhibit a dramatic loss in statistical efficiency, discarding information and essentially converting a "big", information-rich dataset to a much smaller one[7]. Having the ability to fit a large dataset with the same

611 rigorous techniques that work in small datasets would thus represent a major advantage, because of the 612 preservation of statistical efficiency. In our analyses we effortlessly fit a problem that was 4 times as

613 large as the largest one reported in the biomedical literature by fitting the GLMM using the $h$-lik.

614 Although GLMMs are frequently encountered in the analyses of biomedical data, their scope of 615 application is rather broad. For example, an implementation of GLMMs by the Linkedln software engineers[9] showed that the random effect modeling performed among the top 5 to 10 methods in

617 numerous nonmedical datasets used in the KDD Cup competitions. Taken together these results suggest 618 that GLMMs deserve a closer examination by other fields that deal with massive datasets using various 619 ad hoc Machine Learning or Artificial Intelligence approaches.

620 H-lik offers a theoretically rigorous, computational friendly method for estimation of GLMMs

621 The $h$-lik was originally introduced as a unified framework for several common models e.g. Poisson-

622 gamma, binomial-beta with the intention to resolve apparent differences between subject specific and 623 population averaged models[10]. $h$-lik was latter extended to models with structured and unknown 624 dispersion parameters (e.g. the (inverse) Gaussian GLMM) [11] and even the so called "double HGLM" in 625 which random effects can be specified for both mean and variance parameters. The reliance of $h$-lik on 626 the LA would appear to be an area of concern, especially in datasets similar to our own, with few 627 repeated measurements per individual and a binary outcome[38, 39, 59]. Nevertheless, the LA based $h$ 628 lik inferences for fixed and random effects were numerically close to those obtained via the alternative 629 AGH approach. In the case of varying estimates between $h$-lik and AGH, we found the former to be 630 closer to the gold standard of MCMC integration. This observation suggests that higher order LA 
631 introduced in extensions of the basic $h$-lik theory $[11-13,38,39]$ may not be required for the size of 632 datasets considered in this paper. At first, this conclusion appears to contradict previous literature 633 arguing that higher order LA are required to avoid biases in the estimation of variance components[13, $63439,60-64]$. However, this apparent contradiction disappears if one considers the asymptotic order 635 behavior of the LA to integrals over very high dimensional spaces[65]. The error (and thus potential 636 inferential bias) introduced by foregoing corrections is of the order of $o\left(k^{-(1+d) / 2}\right)$, where $d$ is the 637 dimensionality of the space and $k$ a scaling factor of the Hessian at the optimum. Therefore, for analyses 638 of datasets with large number of random effects (large $d$ ), in which the underlying model is reasonably 639 identified by the data (large $k$ ), the approximation error of the LA will decline rapidly to zero because of 640 the "little o" asymptotics. Consequently, while the curse of dimensionality renders higher order AGH 641 approximations impractical, it simultaneously makes the ordinary LA highly accurate in the same 642 settings. Finally, our implementation of the $h$-lik corresponds to method $\mathrm{HL}(1,1)$ in the theory of the 643 hierarchical likelihood, which appears to not be as susceptible to bias as lower order approximations $644[13,39,66,67]$.

645 The $h$-lik approach may be viewed as Empirical Bayes/modal approximations to a non-informative 646 Bayesian analysis of the same data, allowing the analyst to invoke a Bayesian interpretation of the 647 results obtained through the $h$-lik if non-informative priors are appropriate for the problem at hand. In 648 the opinion of the authors, this is a rather significant development since bona fide Bayesian analyses of 649 large datasets via MCMC can be orders of magnitude slower than their likelihood counterparts. Future 650 studies should explore the use (and abuse) of $h$-lik as approximations to full Bayesian analyses in other 651 datasets and define the range of problems and random effects structures for which $h$-lik will give 652 numerical answers that don't differ much from those given by MCMC. A particularly interesting 653 application would explore non-Gaussian random effect distributions, which are trivial to estimate both 654 by the iGLM algorithm and the implementation considered herein. 
655 Scalable alternatives to the proposed $h$-lik implementation, include the R package glmmTMB and the

656 original iGLM algorithm for fitting the hierarchical likelihood approach. glmmTMB was recently

657 introduced [68] for GLMMs and uses an interface similar to R's glmer. However, to our knowledge this

658 package does not implement the $h$-lik approach, although it does appear to be able to estimate non-

659 gaussian GLMMs via REML. The precise definition of the REML implemented in glmmTMB is not

660 explicitly stated in the package documentation. Examination of the R source code of the latter package

661 suggests that REML estimation in gImmTMB amounts to optimization of the $p_{u, \beta}(h)$ function, i.e., the

662 first stage in the hierarchical likelihood approach. Limited comparisons in subsets of CRWD and

663 simulations showed that while the variance components returned by glmmTMB and our approach were

664 identical, estimates of the fixed and random effects could differ, particularly when the number of

665 random effects in the dataset were small. Future work should thus concentrate on investigating

666 alternative ways to optimize the adjusted profile likelihood functions of $h$-lik theory that can offer higher

667 speed advantages to our naïve implementation.

668 The original iGLM approach offers an alternative to the direct implementation of the $h$-lik approach with

669 comparable performance in small datasets. This alternative is also a candidate for a high-performance

670 implementation, since the underlaying operations (iterative solutions of weighted linear regression and

671 numerical optimization) are available in many high-performance scientific computing platforms. Future

672 work may even consider implementing either of the two implementations of the $h$-lik explored in this

673 work within mainstream Machine Learning platforms such as TensorFlow[69]. R research in alternative

674 implementations of the $h$-lik has the potential to make the rigor of GLMMs available to a wider audience 675 of data scientists in addition to statisticians.

676

677 
679 This work was motivated by a clinical question that the authors posed internally a few years ago. We

680 believe that the analysis presented herein answered the question by re-confirming the " $U$ " shaped curve

681 between the $\mathrm{K}^{+}$level and mortality previously suggested [18], but dramatically reducing the uncertainty

682 about this relationship. Based on our data, potassium levels close to the normal level of $4 \mathrm{meq} / \mathrm{l}$ are not

683 associated with excess mortality. Hence, our bracketing of a globally valid reference range for potassium

684 levels could be used to inform the design of clinical trials to guide the proper use of potassium

685 supplements and potassium lowering drugs. The individual and facility level variation detected here is of 686 relevance to future investigations that use EHRs; unless such variation can be captured and modelled

687 through covariates available in the EHRs, inferences from such data will be contaminated by significant 688 bias.

689

690 Conclusions

691 Combining the Laplace Approximation with Automatic Differentiation results in a direct implementation 692 of the $h$-lik that can efficiently fit very large EHR datasets, with accuracy that is equivalent if not better 693 than the state-of-the-art AGH methods and the original iGLM algorithm for GLMMs. For the problems 694 we examined, the h-lik gave results that are indistinguishable from the gold standard of MCMC 695 integration, but the results were obtained much faster.

\section{List of abbreviations:}

697 AD: Algorithmic Differentiation

698 AGH: Adaptive Gaussian Hermite Quadrature

699 ANOVA: Analysis of Variance

700 BLAS: Basic Linear Algebra Subprograms 
701 BLUP: Best Linear Unbiased Predictor

702 CRWD: Cerner Real World Data

703 eGFR: Estimated Glomerular Filtration Rate

704 EHR: Electronic Health Record

705 EQL: Extended Quasi Likelihood

706 GLM: Generalized Linear Model

707 GLMM: Generalized Linear Mixed Model

708 HCP: Healthcare Practitioners

709 HCF: Healthcare Facilities

710 h-lik: Hierarchical Likelihood

$711 \quad \mathbf{K}^{+}$: Serum potassium level

712 iGLM: Interconnected Generalized Linear Models

713 IWLS: Iterative Weighted Linear Squares

714 IP: Individual Patients

715 LA: Laplace Approximation

716 MCMC: Markov Chain Monte Carlo

717 MLE: Maximum Likelihood Estimation

718 REML: Restricted Maximum Likelihood 


\section{Declarations:}

720 Ethics approval and consent to participate: Analyses with CRWD are considered "Not Human Research"

721 by the Human Research Protection Office (ethics committee and Institutional Review Board) of the

722 University of New Mexico Health Sciences Center

723 Consent for publication: Not applicable

724 Availability of data and materials: The data that support the findings of this study are available from

725 Cerner Corporation, but restrictions apply to the availability of these data, which were used under

726 license for the current study, and so are not publicly available. The Cerner Health Facts Database (now

727 referred to as the Cerner Real World Data, CRWD) is available to researchers at contributing hospitals

728 (and their research affiliates) upon request made directly to Cerner Corporation. Software code for the

729 implementation of the LA for a Poisson regression with one and two random effects is available from the

730 repository: https://bitbucket.org/chrisarg/laplaceapproximationandhyperkalemia/

731 Competing interests: CA has received consulting fees from Bayer, Baxter Healthcare, Health Services

732 Advisory Group and research support from Dialysis Clinic, Inc. CGB, VSP MLU, MER, VS, SKS, SA have

733 received support from Dialysis Clinic, Inc. JC has nothing to disclose.

734 Funding: This research was funded in part by Dialysis Clinic Inc under grant RF\#C-4011

735 Author's contributions: CB extracted and preprocessed the data from the CRWD database used in this

736 analysis, VSP, JC and CA designed the comparative evaluations used in the manuscript and programmed

737 the analysis in R. MLU, MER, VS, SKS, SA and CA interpreted the clinical analyses of hyperkalemia and

738 the risk of death.

739 Acknowledgements: Not applicable 


\section{References:}

742 1. Bates DW, Saria S, Ohno-Machado L, Shah A, Escobar G. Big Data In Health Care: Using Analytics To 743 Identify And Manage High-Risk And High-Cost Patients. Health Affairs. 2014;33:1123-31.

744

745

746

747

748

749

750

751

752

753

754

755

756

757

758

759

760

761

762

763

764

765

766

767

768

769

770

771

772

773

774

2. Goldstein BA, Navar AM, Pencina MJ, loannidis JPA. Opportunities and challenges in developing risk prediction models with electronic health records data: a systematic review. J Am Med Inform Assoc. 2017;24:198-208.

3. Krumholz HM. Big Data And New Knowledge In Medicine: The Thinking, Training, And Tools Needed For A Learning Health System. Health Affairs. 2014;33:1163-70.

4. Silverio A, Cavallo P, De Rosa R, Galasso G. Big Health Data and Cardiovascular Diseases: A Challenge for Research, an Opportunity for Clinical Care. Front Med (Lausanne). 2019;6. doi:10.3389/fmed.2019.00036.

5. Rajkomar A, Oren E, Chen K, Dai AM, Hajaj N, Hardt M, et al. Scalable and accurate deep learning with electronic health records. npj Digital Med. 2018;1:1-10.

6. Gebregziabher M, Egede L, Gilbert GE, Hunt K, Nietert PJ, Mauldin P. Fitting parametric random effects models in very large data sets with application to VHA national data. BMC Medical Research Methodology. 2012;12:163.

7. Perry PO. Fast moment-based estimation for hierarchical models. Journal of the Royal Statistical Society: Series B (Statistical Methodology). 2017;79:267-91.

8. Lee JYL, Brown JJ, Ryan LM. Sufficiency Revisited: Rethinking Statistical Algorithms in the Big Data Era. The American Statistician. 2017;71:202-8.

9. Zhang X, Zhou Y, Ma Y, Chen B-C, Zhang L, Agarwal D. GLMix: Generalized Linear Mixed Models For Large-Scale Response Prediction. In: Proceedings of the 22Nd ACM SIGKDD International Conference on Knowledge Discovery and Data Mining. New York, NY, USA: ACM; 2016. p. 363-72. doi:10.1145/2939672.2939684.

10. Lee $Y$, Nelder JA. Hierarchical Generalized Linear Models. Journal of the Royal Statistical Society Series B (Methodological). 1996;58:619-78.

11. Lee Y, Nelder JA. Hierarchical Generalised Linear Models: A Synthesis of Generalised Linear Models, Random-Effect Models and Structured Dispersions. Biometrika. 2001;88:987-1006.

12. Lee Y, Nelder JA, Pawitan Y. Generalized Linear Models with Random Effects: Unified Analysis via Hlikelihood, Second Edition. 2 edition. Boca Raton, Florida: Chapman and Hall/CRC; 2017.

13. Collins D. The performance of estimation methods for generalized linear mixed models. University of Wollongong; 2008. https://ro.uow.edu.au/theses/1737.

14. Kristensen K, Nielsen A, Berg CW, Skaug H, Bell BM. TMB: Automatic Differentiation and Laplace Approximation. Journal of Statistical Software. 2016;70. doi:10.18637/jss.v070.i05. 
15. Skaug HJ. Automatic Differentiation to Facilitate Maximum Likelihood Estimation in Nonlinear Random Effects Models. Journal of Computational and Graphical Statistics. 2002;11:458-70.

16. Skaug HJ, Fournier DA. Automatic approximation of the marginal likelihood in non-Gaussian hierarchical models. Computational Statistics \& Data Analysis. 2006;51:699-709.

17. Nilsson E, Gasparini A, Ärnlöv J, Xu H, Henriksson KM, Coresh J, et al. Incidence and determinants of hyperkalemia and hypokalemia in a large healthcare system. International Journal of Cardiology. 2017;245:277-84.

18. Luo J, Brunelli SM, Jensen DE, Yang A. Association between Serum Potassium and Outcomes in Patients with Reduced Kidney Function. CJASN. 2016;11:90-100.

19. Chapman Neil, Dobson Joanna, Wilson Sarah, Dahlöf Björn, Sever Peter S., Wedel Hans, et al. Effect of Spironolactone on Blood Pressure in Subjects With Resistant Hypertension. Hypertension. 2007;49:839-45.

20. Zannad F, McMurray JJV, Krum H, van Veldhuisen DJ, Swedberg K, Shi H, et al. Eplerenone in Patients with Systolic Heart Failure and Mild Symptoms. http://dx.doi.org/10.1056/NEJMoa1009492. 2011. doi:10.1056/NEJMoa1009492.

21. Pitt B, Zannad F, Remme WJ, Cody R, Castaigne A, Perez A, et al. The Effect of Spironolactone on Morbidity and Mortality in Patients with Severe Heart Failure. http://dx.doi.org/10.1056/NEJM199909023411001. 2008. doi:10.1056/NEJM199909023411001.

22. Linde C, Qin L, Bakhai A, Furuland H, Evans M, Ayoubkhani D, et al. Serum potassium and clinical outcomes in heart failure patients: results of risk calculations in 21334 patients in the UK. ESC Heart Fail. 2019;6:280-90.

23. Bakris GL, Agarwal R, Anker SD, Pitt B, Ruilope LM, Rossing P, et al. Effect of Finerenone on Chronic Kidney Disease Outcomes in Type 2 Diabetes. New England Journal of Medicine. 2020;0:null.

24. Bolignano D, Palmer SC, Navaneethan SD, Strippoli GFM. Aldosterone antagonists for preventing the progression of chronic kidney disease. Cochrane Database Syst Rev. 2014;:CD007004.

25. Trevisan M, de Deco P, Xu H, Evans M, Lindholm B, Bellocco R, et al. Incidence, predictors and clinical management of hyperkalaemia in new users of mineralocorticoid receptor antagonists. Eur J Heart Fail. 2018;20:1217-26.

26. Bologa C, Pankratz VS, Unruh ML, Roumelioti ME, Shah V, Shaffi SK, et al. Generalized Mixed Modeling in Massive Electronic Health Record Databases: what is a healthy serum potassium? arXiv:191008179 [stat]. 2019. http://arxiv.org/abs/1910.08179. Accessed 16 Nov 2020.

27. Argyropoulos C, Bologa C George, Pankratz VS, Unruh ML, Roumelioti ME, Shah V, et al. Association of Potassium Level and Mortality in Massive Health Record Databases. In: Journal of The American Society of Nephrology. San Diego; 2018. p. 499-500.

28. Argyropoulos C, Unruh ML. Analysis of Time to Event Outcomes in Randomized Controlled Trials by Generalized Additive Models. PLoS ONE. 2015;10:e0123784. 
29. Liu Q, Pierce DA. A Note on Gauss-Hermite Quadrature. Biometrika. 1994;81:624-9.

812 30. Pinheiro JC, Bates DM. Approximations to the Log-Likelihood Function in the Nonlinear Mixed-Effects 813 Model. Journal of Computational and Graphical Statistics. 1995;4:12-35.

814 31. Pinheiro JC, Bates DM. Mixed Effects Models in S and S-Plus. Springer; 2000.

815 32. Pinheiro JC, Chao EC. Efficient Laplacian and Adaptive Gaussian Quadrature Algorithms for Multilevel 816 Generalized Linear Mixed Models. Journal of Computational and Graphical Statistics. 2006;15:58-81.

817 33. Liu Q. Laplace approximations to likelihood functions for generalized linear mixed models. 1994. 818 https://ir.library.oregonstate.edu/concern/graduate_thesis_or_dissertations/b8515r59n?locale=en.

819 34. Wolfinger R. Laplace's Approximation for Nonlinear Mixed Models. Biometrika. 1993;80:791-5.

820 35. Skinner L. Note on the Asymptotic Behavior of Multidimensional Laplace Integrals. SIAM J Math Anal. 821 1980;11:911-7.

822 36. Capanu M, Gönen M, Begg CB. An assessment of estimation methods for generalized linear mixed 823 models with binary outcomes. Statist Med. 2013;32:4550-66.

824 37. McGilchrist CA, Yau KKW. The derivation of blup, ML, REML estimation methods for generalised 825 linear mixed models. Communications in Statistics - Theory and Methods. 1995;24:2963-80.

826 38. Noh M, Lee Y. REML estimation for binary data in GLMMs. Journal of Multivariate Analysis. $827 \quad 2007 ; 98: 896-915$.

828 39. Lee W, Lee Y. Modifications of REML algorithm for HGLMs. Stat Comput. 2012;22:959-66.

829 40. Cox DR, Reid N. Parameter Orthogonality and Approximate Conditional Inference. Journal of the 830 Royal Statistical Society: Series B (Methodological). 1987;49:1-18.

831 41. Lee Y, Nelder JA. Double hierarchical generalized linear models (with discussion). Journal of the Royal 832 Statistical Society: Series C (Applied Statistics). 2006;55:139-85.

833 42. Nelder JA, Pregibon D. An Extended Quasi-Likelihood Function. Biometrika. 1987;74:221-32.

834 43. Rönnegård L, Shen X, Alam M. hglm: A Package for Fitting Hierarchical Generalized Linear Models. 835 The R Journal. 2010;2:20-8.

836 44. Molas M, Lesaffre E. Hierarchical Generalized Linear Models: The R Package HGLMMM. Journal of 837 Statistical Software. 2011;39:1-20.

838 45. Beck A, Tetruashvili L. On the Convergence of Block Coordinate Descent Type Methods. SIAM J 839 Optim. 2013;23:2037-60.

840 46. Lange K, Chi EC, Zhou H. A Brief Survey of Modern Optimization for Statisticians: Modern 841 Optimization for Statisticians. International Statistical Review. 2014;82:46-70. 
842 47. Griewank Andreas, Walther Andrea. Evaluating Derivatives. Society for Industrial and Applied 843 Mathematics; 2008. doi:10.1137/1.9780898717761.

844 48. Bartholomew-Biggs M, Brown S, Christianson B, Dixon L. Automatic differentiation of algorithms. 845 Journal of Computational and Applied Mathematics. 2000;124:171-90.

846 49. Baydin AG, Pearlmutter BA, Radul AA, Siskind JM. Automatic Differentiation in Machine Learning: a 847 Survey. Journal of Machine Learning Research. 2018;18:1-43.

848 50. Gebremedhin AH, Manne F, Pothen A. What Color Is Your Jacobian? Graph Coloring for Computing 849 Derivatives. SIAM Review. 2005;47:629-705.

850 51. Coleman TF, Moré JJ. Estimation of sparse hessian matrices and graph coloring problems.

851 Mathematical Programming. 1984;28:243-70.

852 52. Blackford LS, Petitet A, Pozo R, Remington K, Whaley RC, Demmel J, et al. An updated set of basic 853 linear algebra subprograms (BLAS). ACM Transactions on Mathematical Software. 2002;28:135-51.

854 53. Meng X-L. Decoding the H-likelihood. Statist Sci. 2009;24:280-93.

855 54. Bates D, Mächler M, Bolker B, Walker S. Fitting Linear Mixed-Effects Models Using Ime4. Journal of 856 Statistical Software. 2015;67:1-48.

857 55. Stan Development Team. Stan Modeling Language Users Guide and Reference Manual, Version 2.25. 858 2019. http://mc-stan.org/.

859 56. Stan Development Team. RStan: the R interface to Stan. 2020. http://mc-stan.org/.

860 57. Hoffman MD, Gelman A. The No-U-Turn Sampler: Adaptively Setting Path Lengths in Hamiltonian 861 Monte Carlo. Journal of Machine Learning Research. 2014;15:1593-623.

862 58. Ruppert D, Wand MP, Carroll RJ. Semiparametric regression during 2003-2007. Electron J Stat. $863 \quad 2009 ; 3: 1193-256$.

864 59. Vonesh EF. A Note on the Use of Laplace's Approximation for Nonlinear Mixed-Effects Models. 865 Biometrika. 1996;83:447-52.

866 60. Breslow NE, Lin X. Bias Correction in Generalised Linear Mixed Models with a Single Component of 867 Dispersion. Biometrika. 1995;82:81-91.

868 61. Lin X, Breslow NE. Bias Correction in Generalized Linear Mixed Models With Multiple Components of 869 Dispersion. Journal of the American Statistical Association. 1996;91:1007-16.

870 62. Shun Z. Another Look at the Salamander Mating Data: A Modified Laplace Approximation Approach. 871 Journal of the American Statistical Association. 1997;92:341-9.

872 63. Shun Z, McCullagh P. Laplace Approximation of High Dimensional Integrals. Journal of the Royal 873 Statistical Society Series B (Methodological). 1995;57:749-60. 
874 64. Noh M, Lee Y. REML estimation for binary data in GLMMs. Journal of Multivariate Analysis.

$875 \quad 2007 ; 98: 896-915$.

876 65. Kirwin WD. Higher asymptotics of Laplace's approximation. Asymptotic Analysis. 2010;70:231-48.

877 66. Yun S, Lee Y. Comparison of hierarchical and marginal likelihood estimators for binary outcomes.

878 Computational Statistics \& Data Analysis. 2004;45:639-50.

879 67. Lee W, Lim J, Lee Y, del Castillo J. The hierarchical-likelihood approach to autoregressive stochastic 880 volatility models. Computational Statistics \& Data Analysis. 2011;55:248-60.

881 68. Brooks ME, Kristensen K, Benthem KJ van, Magnusson A, Berg CW, Nielsen A, et al. glmmTMB

882 Balances Speed and Flexibility Among Packages for Zero-inflated Generalized Linear Mixed Modeling.

883 The R Journal. 2017;9:378-400.

884 69. Martín Abadi, Ashish Agarwal, Paul Barham, Eugene Brevdo, Zhifeng Chen, Craig Citro, et al.

885 TensorFlow: Large-Scale Machine Learning on Heterogeneous Systems. 2015.

886 https://www.tensorflow.org/. 


\section{Figure Legends}

890 Figure 1: Estimates and 95\% confidence intervals for the fixed effect components

891 Figure 2: Estimated cubic splines relating the relative risk of death to potassium level and their associated $89295 \%$ confidence $(h$-lik) and credible (MCMC) intervals using the $1 \%$ dataset and a single random effect at 893 the healthcare facility level

894 Figure 3: Comparison of the predicted random effects (BLUPs) as estimated by the $h$-lik, the various AGH 895 and the MCMC methods

896 Figure 4: GLMM execution timings for different datasets for single random effects models, either at the 897 individual patient or the healthcare facility level

898 Figure 5: Estimated cubic splines relating the relative risk of death to potassium level and their associated $89995 \%$ confidence by the direct $h$-lik implementation for various dataset sizes. Poisson models included 900 random effects at both the individual and the healthcare facility levels (gray lines demarcate the zone in 901 which RR is between 0.9 and 1.1).

902 Figure 6: Performance (total wall time) of $h$-lik computations using serial and parallel codes.

903 Figure 7: Execution times of the steps of $h$-lik calculations for serial and parallel codes. 
Figures

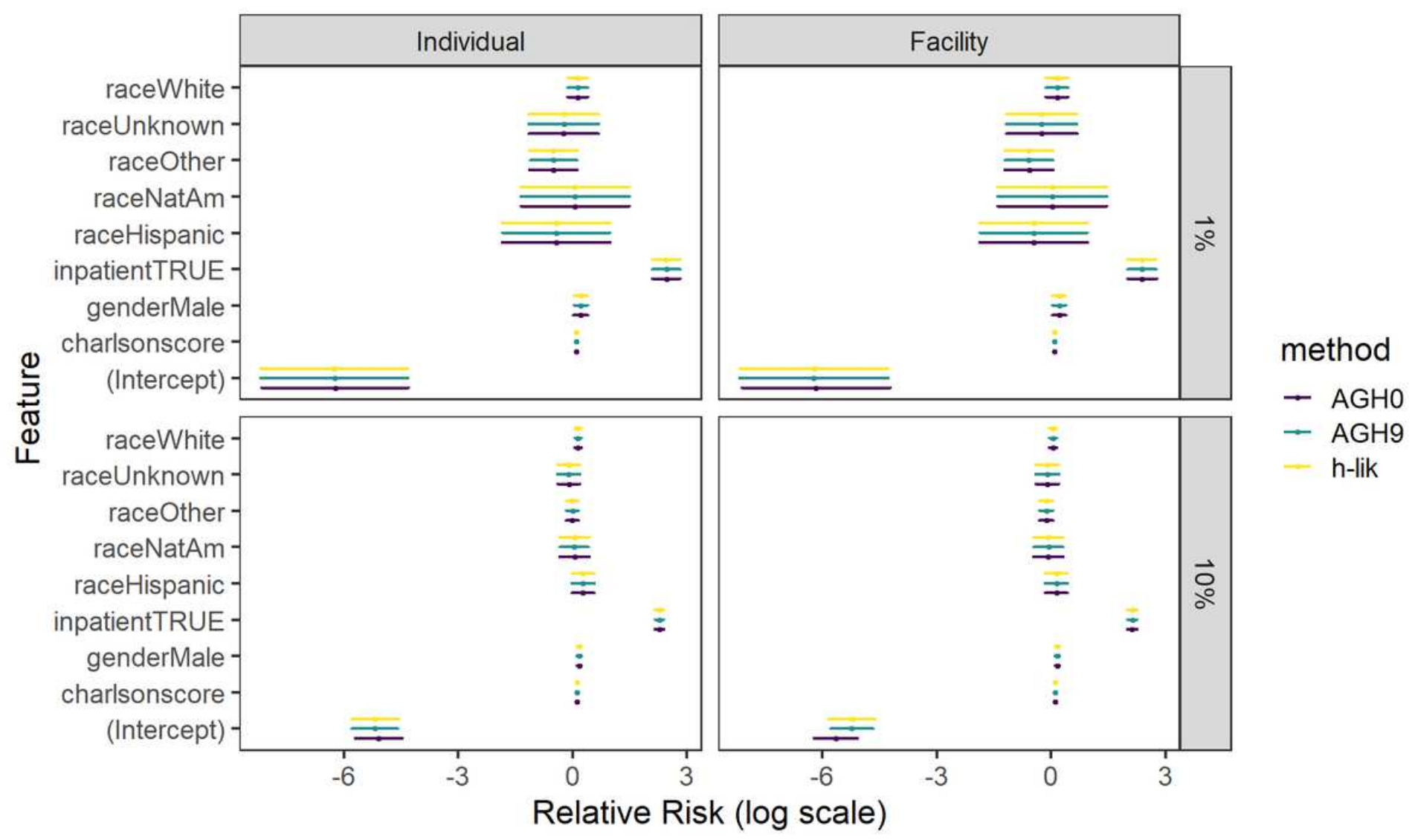

\section{Figure 1}

Estimates and $95 \%$ confidence intervals for the fixed effect components 


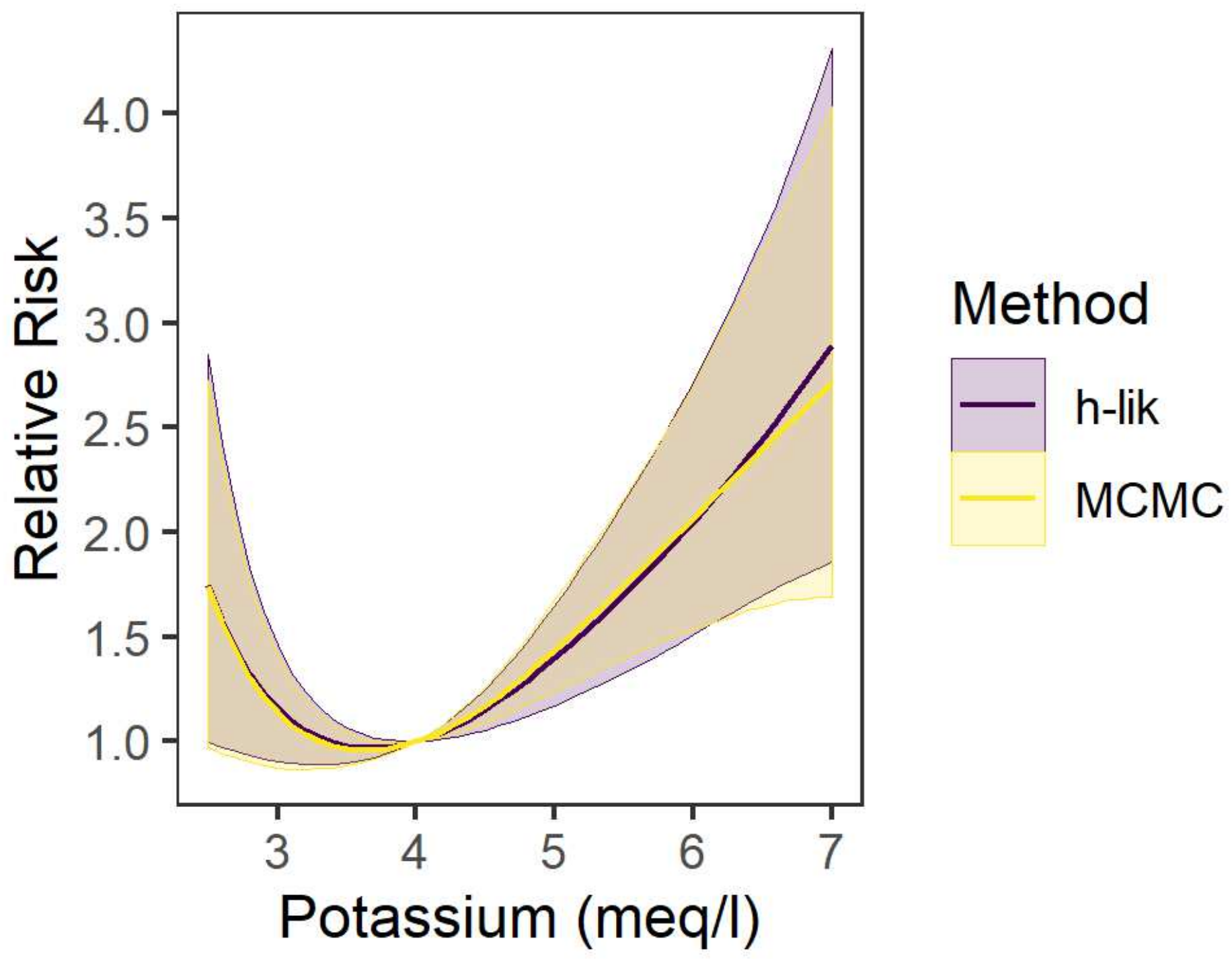

Figure 2

Estimated cubic splines relating the relative risk of death to potassium level and their associated $95 \%$ confidence (h-lik) and credible (MCMC) intervals using the 1\% dataset and a single random effect at the healthcare facility level 

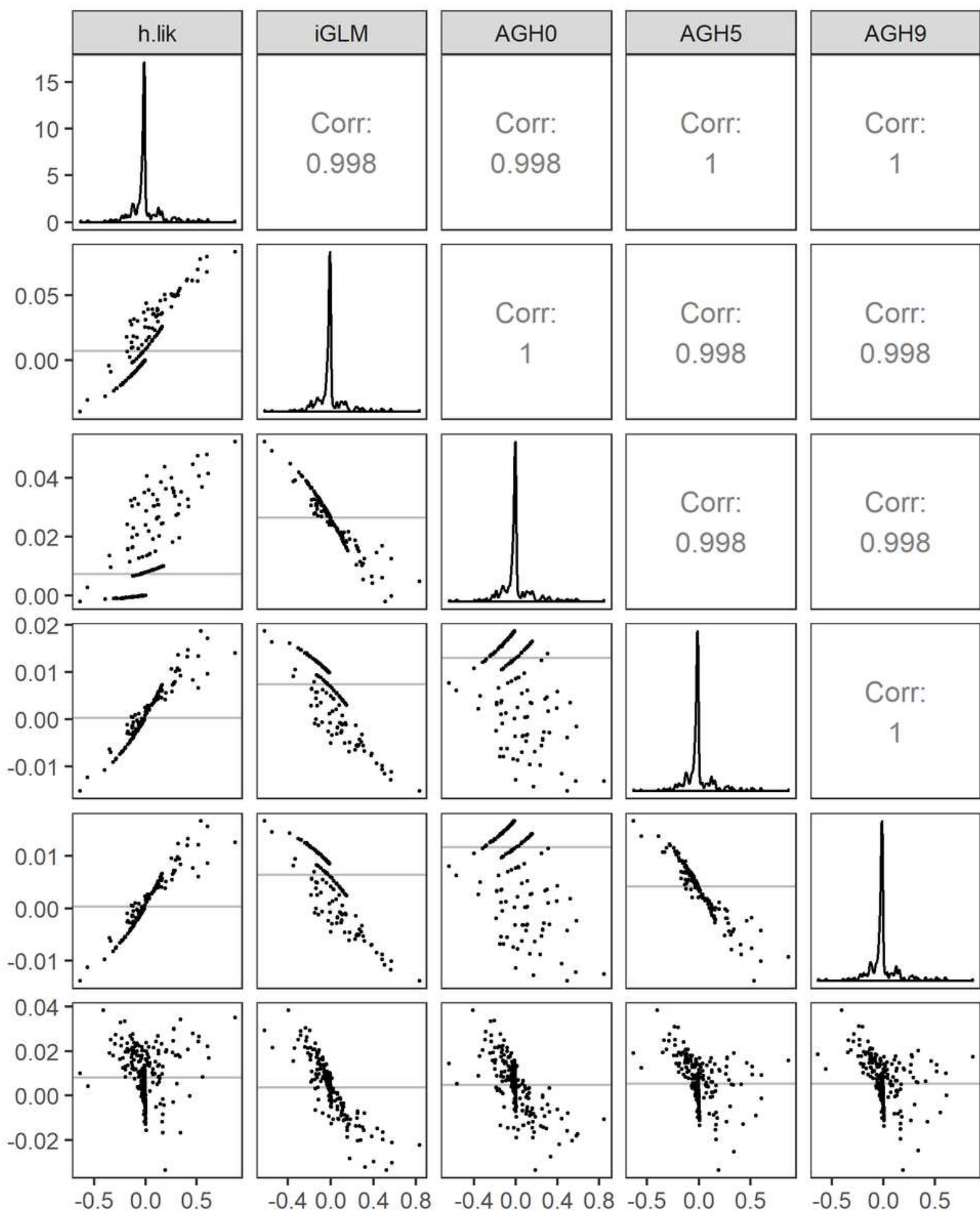
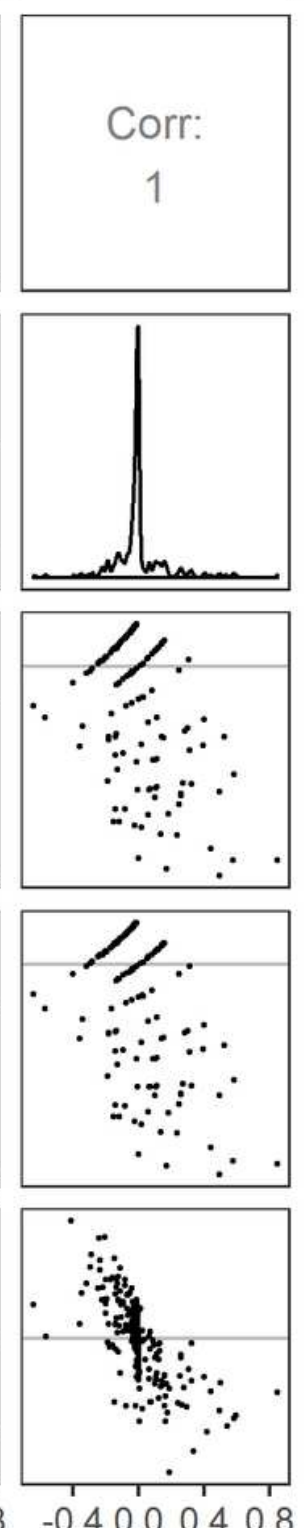
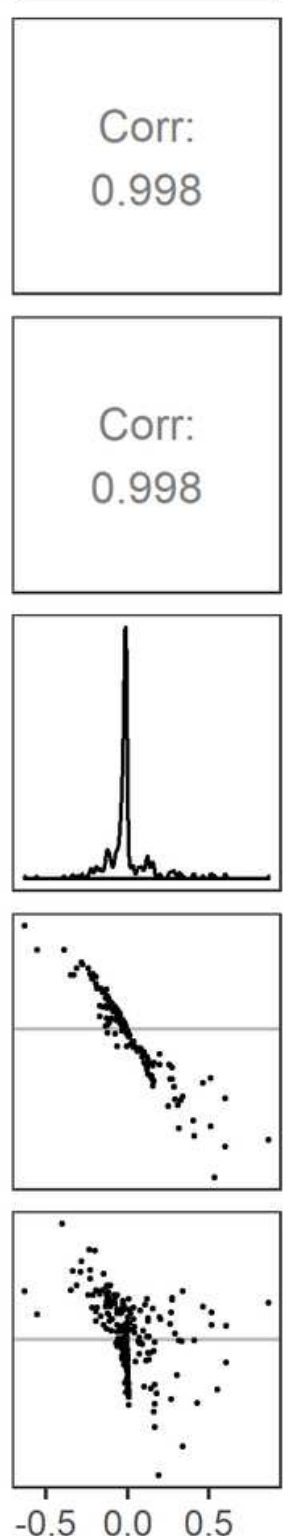
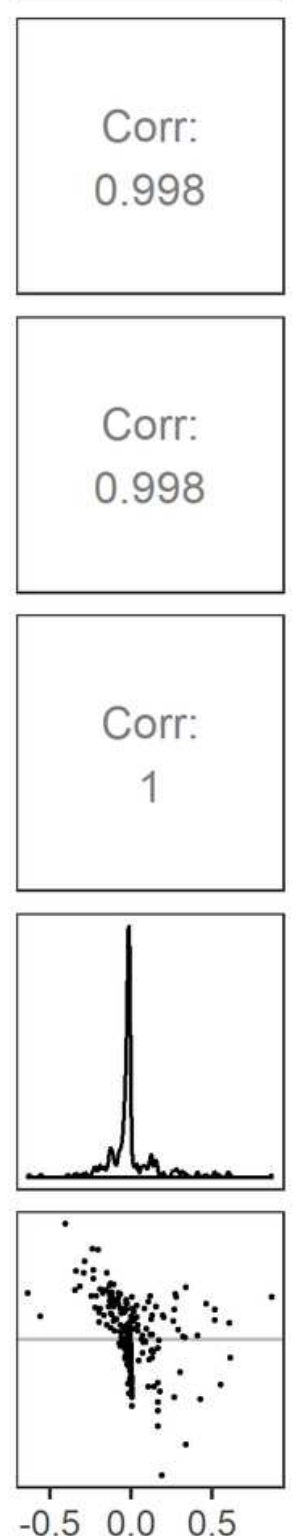
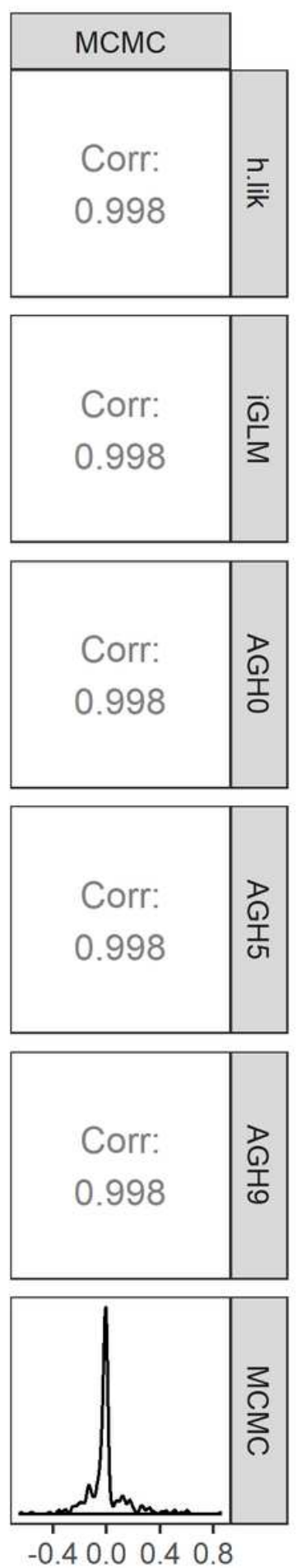

Figure 3

Comparison of the predicted random effects (BLUPs) as estimated by the h-lik, the various AGH and the MCMC methods 


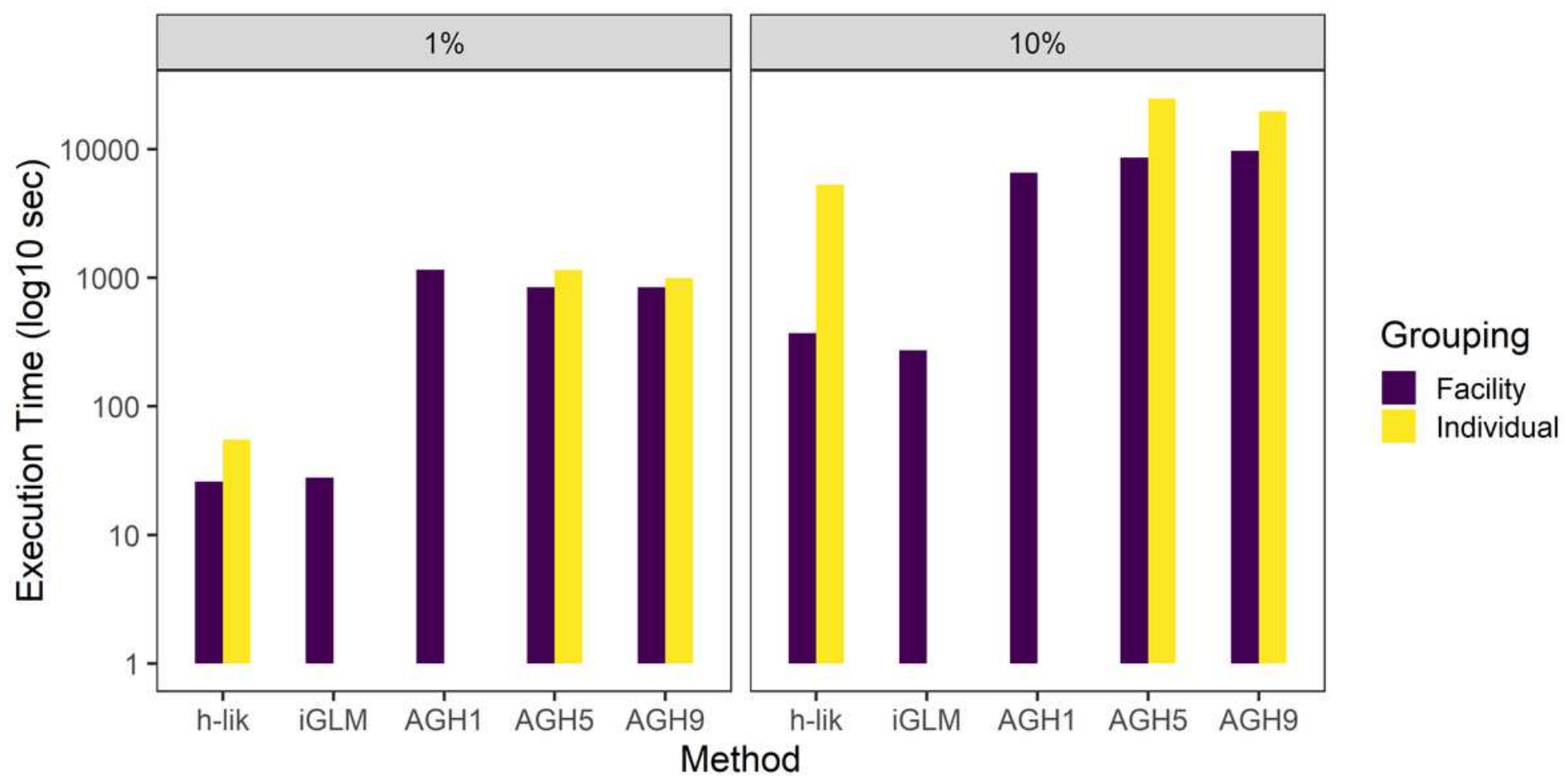

Figure 4

GLMM execution timings for different datasets for single random effects models, either at the individual patient or the healthcare facility level 


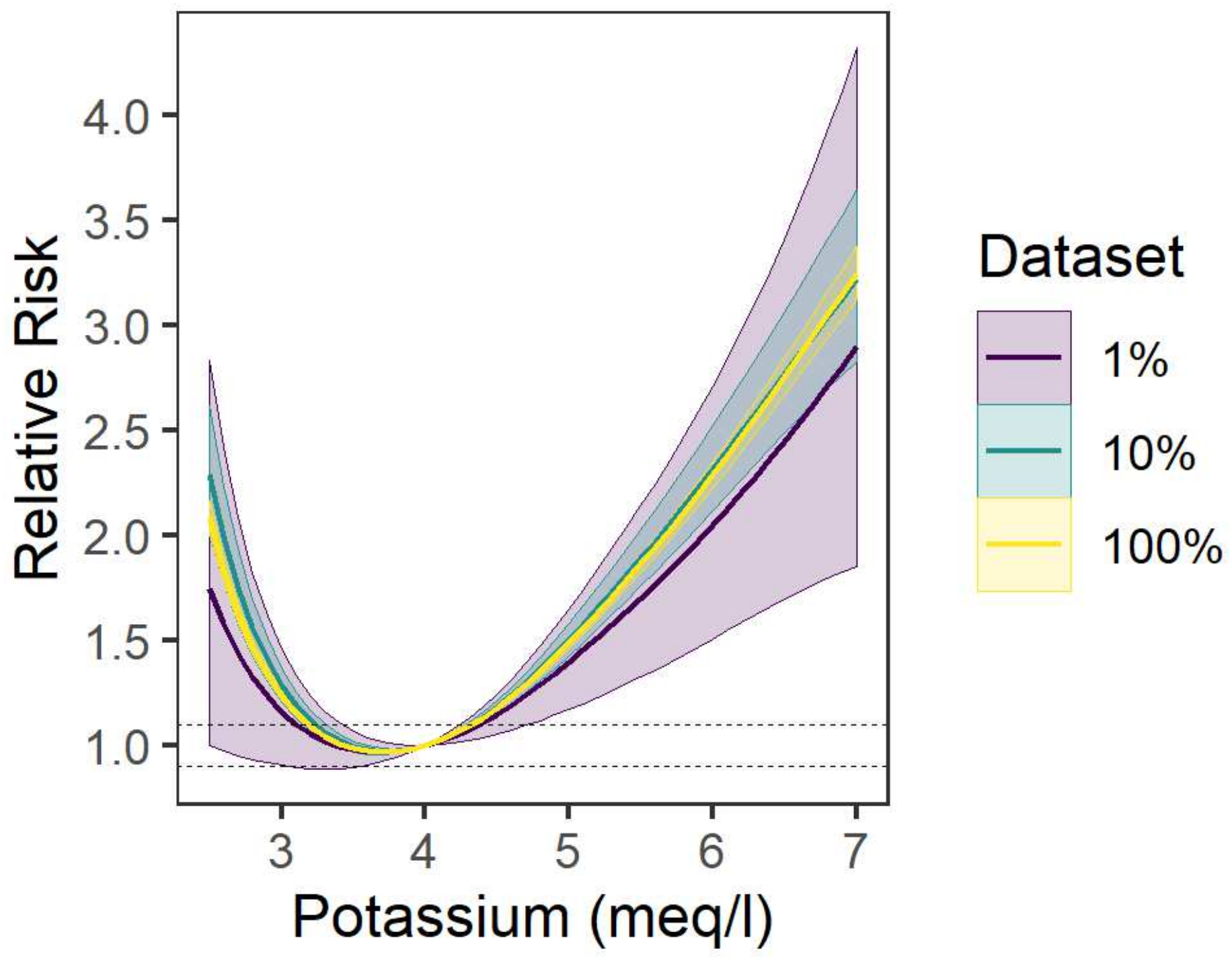

Figure 5

Estimated cubic splines relating the relative risk of death to potassium level and their associated $95 \%$ confidence by the direct h-lik implementation for various dataset sizes. Poisson models included random effects at both the individual and the healthcare facility levels (gray lines demarcate the zone in which RR is between 0.9 and 1.1). 


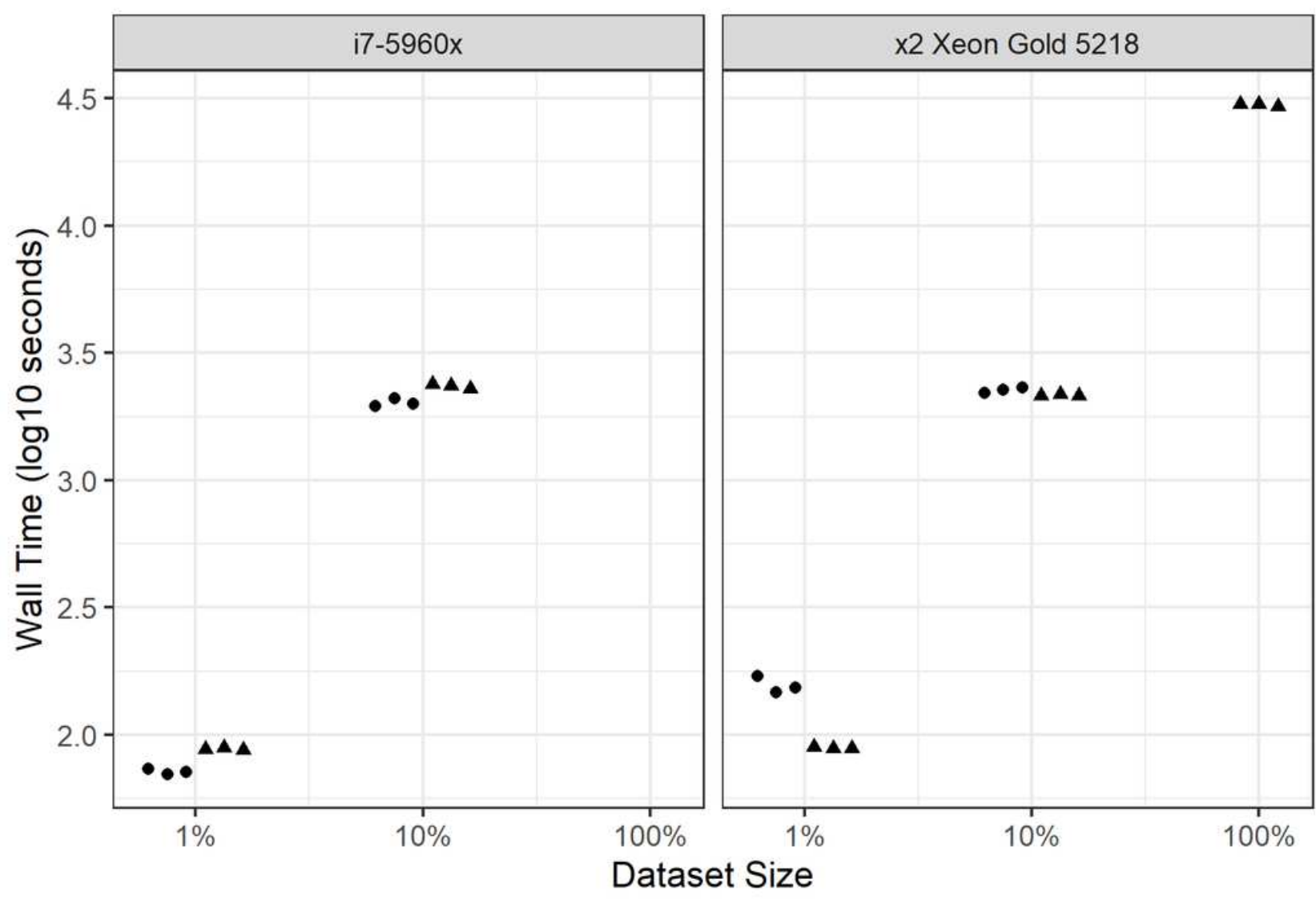

Code

- Parallel

- Serial

Figure 6

Performance (total wall time) of h-lik computations using serial and parallel codes. 

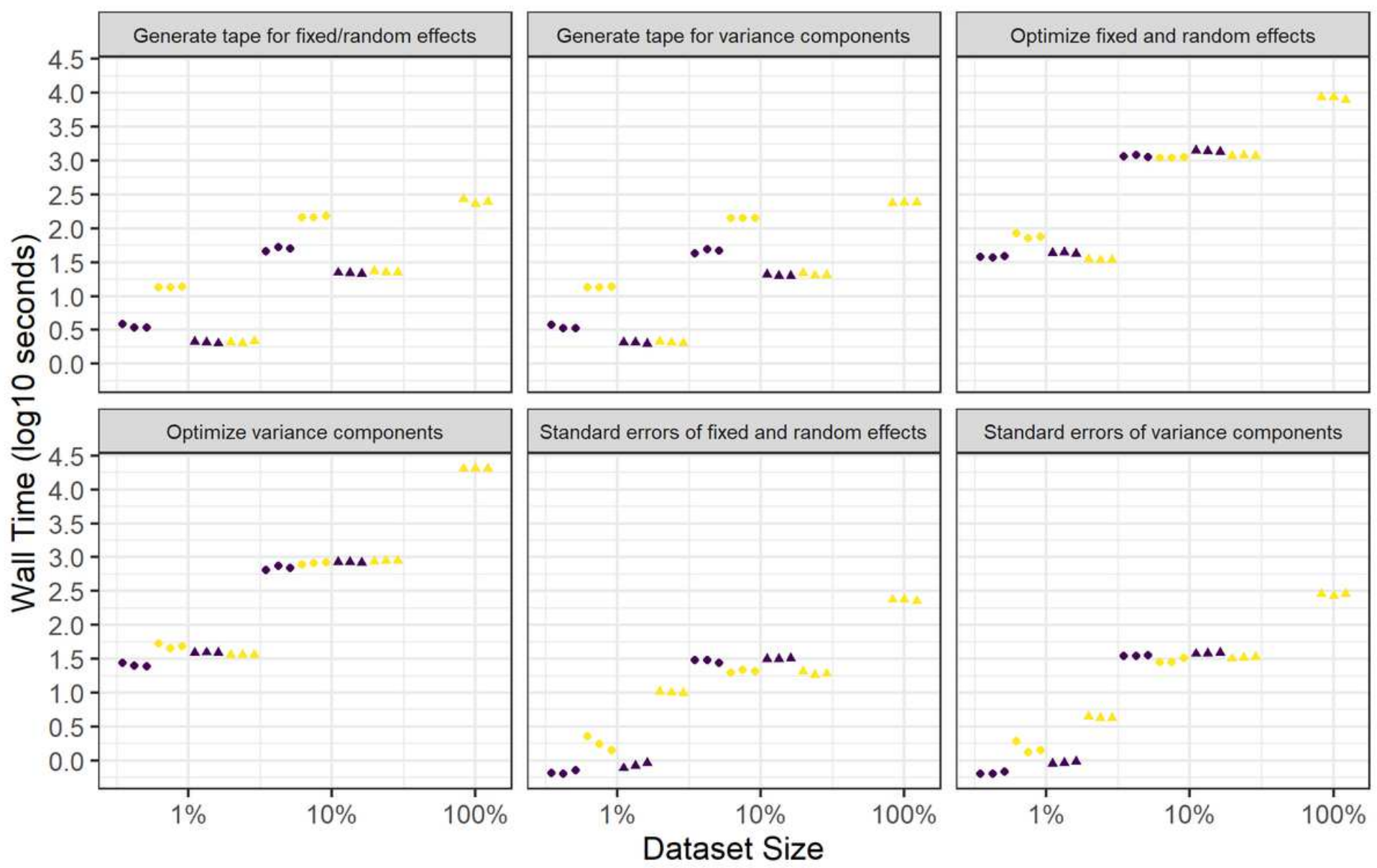

Code - Parallel A Serial Machine - i7-5960x ×2 Xeon Gold 5218

Figure 7

Execution times of the steps of h-lik calculations for serial and parallel codes. 